\title{
In vivo Observations of Pre- and Postsynaptic Changes during the Transition from Multiple to Single Innervation at Developing Neuromuscular Junctions
}

\author{
Rita J. Balice-Gordon and Jeff W. Lichtman \\ Department of Anatomy and Neurobiology, Washington University School of Medicine, St. Louis, Missouri 63110
}

Synaptic rearrangements in developing muscle were studied by visualizing individual neuromuscular junctions in the sternomastoid muscle of living neonatal mice as they underwent the transition from multiple to single innervation. Vital staining of $\mathrm{ACh}$ receptors (AChRs) with rhodamine-conjugated $\alpha$-bungarotoxin showed that while junctions were still multiply innervated (usually by two motor axons), regions of the postsynaptic membrane within each junction became depleted of receptors. Usually, several small postsynaptic areas lost AChRs in succession. In these areas, AChRs already in the membrane rapidly disappeared compared to a low level of receptor turnover elsewhere in the junction. Moreover, there was no evidence of new AChRs being inserted into these areas. Within each postsynaptic area undergoing AChR depletion, the intensity of receptor staining decreased gradually over 1-2 d. In some junctions, it appeared that AChRs were migrating away from areas being depleted of receptors. The depletion of AChRs from some sites in combination with the spreading apart of the entire receptor-rich area due to muscle fiber growth accounts for the transformation from plaque-like to branched receptor distributions at developing neuromuscular junctions.

Vital staining of presynaptic motor nerve terminals at junctions whose postsynaptic AChRs were also stained showed that motor nerve terminals were lost from the same areas that were depleted of receptors postsynaptically. Postsynaptic areas began to be depleted of AChRs before there was any obvious loss of membrane or intracellular staining in the overlying nerve terminal. Only when a single innervating axon remained at a junction did loss of motor nerve terminals and underlying AChRs largely cease. That former synaptic areas could at later times be identified as uninnervated regions within a junction indicates that synapse elimination during development leaves an indelible mark on synaptic structure.

These observations suggest that the withdrawal of a motor axon from a neuromuscular junction occurs as a consequence of the stepwise elimination of all of its synapses with that muscle fiber. These results also suggest that an im-

\footnotetext{
Received July 15, 1992; revised Aug. 21, 1992; accepted Aug. 28, 1992.

We thank A. Brown, H. Colman, S. Culican, K. Fischer, C. Nelson, M. Rich, S. Turney, and P. van Mier for helpful discussions and C. Chua, J. Fiez, L. Hedayati, and S. Turney for technical assistance. This work was supported by grants from the NIH and MDA to J.W.L. and the Harry Zimmerman Postdoctoral Fellowship of the MDA to R.B.-G.

Correspondence should be addressed to Rita J. Balice-Gordon, Ph.D., Department of Anatomy and Neurobiology, Washington University School of Medicine, 660 South Euclid Avenue, Box 8108, St. Louis, MO 63110.

Copyright (C) 1993 Society for Neuroscience $0270-6474 / 93 / 130834-22 \$ 05.00 / 0$
}

portant aspect of synaptic competition leading to axon withdrawal is the precocious loss of AChRs beneath the nerve terminals of the axon that will be eliminated. A similar early loss of AChRs beneath one axon's synapses has been shown to occur during synapse elimination in reinnervated adult muscle (Rich and Lichtman, 1989a). Thus, changes in the postsynaptic membrane beneath the nerve terminals of one motor axon may instigate the removal of those terminals, suggesting that the postsynaptic cell plays an important role in competitive synaptic reorganization.

[Key words: synapse elimination, competition, neuromuscular junction, ACh receptors, $\alpha$-bungarotoxin, nerve terminals, fluorescent dyes, vital dyes, motor neuron, axon, muscle fiber]

Throughout the developing nervous system, competitive interactions between different neurons result in extensive and permanent changes in the pattern of synaptic connections. For example, in the developing visual system, inputs driven by the two eyes are initially convergent, innervating the same target cells in primary visual cortex (Hubel and Wiesel, 1963). After birth, however, these inputs apparently compete for domination of the same cortical neurons. As a result, inputs driven by the two eyes segregate onto different cortical neurons by the elimination of synaptic connections established earlier (LeVay et al., 1980). This rearrangement results in the formation of ocular dominance columns that are permanently maintained. Because the process of segregation is profoundly affected when the visual experience of the two eyes is unequal, the neural activity of the competing inputs appears to have an important influence on the outcome (Hubel and Wiesel, 1970). Activity-driven synaptic rearrangements may also be involved in other long-term changes in the nervous system, for example, in learning and memory.

Few details are known about the mechanism of synaptic competition. The complexity and inaccessibility of synaptic circuitry in the CNS have made it difficult to study this phenomenon at the level of individual synapses. However, a similar competitive reorganization of synapses also occurs in the more accessible periphery, in both muscle (Redfern, 1970; Brown et al., 1976; see Jansen and Fladby, 1990, for recent review) and autonomic ganglia (Lichtman, 1977; see Purves and Lichtman, 1980, for review). In developing mammalian muscle, for example, several different motor axons converge at the same neuromuscular junction on each muscle fiber. During the first weeks after birth, some motor axon branches are eliminated, pruning each motor unit and leaving each muscle fiber innervated by a single motor neuron. This process is commonly called synapse elimination. 
We have studied synapse elimination by repeatedly observing the same junctions over time in the sternomastoid muscle of living mice. In this muscle, most junctions are innervated by two axons at birth but by only one axon 2 weeks later (Steinbach, 1981). One of our aims was to determine whether axon withdrawal occurs abruptly, with the sudden demise of all of an axon's synapses, as has been suggested by some experiments (Rosenthal and Taraskevitch, 1977). Alternatively, axon withdrawal may be the outcome of a protracted competition between two axons that leads to the gradual loss of territory by one axon. Our evidence suggests that withdrawal of the losing motor axon from a junction is the culmination of a gradual process of synaptic loss. Another aim was to study the role that the postsynaptic muscle fiber might play in this process. Previous work in adult muscle showed that after muscle nerve crush and reinnervation, terminal sprouts that transiently multiply innervated junctions regressed over time (Rich and Lichtman, 1989a). As sprouts were eliminated, postsynaptic ACh receptors (AChRs) were observed to be lost from the postsynaptic membrane at former sites of synaptic contact. In fact, AChR loss preceded nerve terminal loss. We were interested to learn if a similar relation held for postsynaptic sites that lose nerve terminals during development.

Steinbach (1981) and Slater (1982a,b) have noted that the AChR distribution within neuromuscular junctions changes dramatically during the first few postnatal weeks when multiple innervation is being eliminated. Whereas at birth the AChRs in each neuromuscular junction are typically arranged as ovalshaped plaques with only hints of inhomogeneities in receptor density, by 2-3 weeks postnatal the AChR areas have been transformed into a more mature pattern with regions of high and very low receptor density. The high-density areas become the sites of the primary junctional folds that are overlain by nerve terminal branches, while the low-density sites are not innervated. Although these workers did not view the same junctions multiple times, they argued that the transition from plaque to mature forms was accomplished by a disappearance of AChRs from areas within the plaques. This idea was supported by the presence of "transitional" junctions in early postnatal ages in which AChR-rich plaques contained onc or more small holes with low receptor density (i.e., "perforated plaques"; Nyström, 1968). Could it be that during development AChR-rich areas are lost as part of the synapse elimination process? In the present work, we determined that postsynaptic changes in $\mathrm{AChR}$ density do occur at sites of nerve terminal elimination. Moreover, postsynaptic AChR depletion precedes the loss of overlying nerve terminals as previously found in reinnervated adult junctions, arguing for the importance of the postsynaptic cell as an intermediary in synaptic competition during normal development.

A portion of these results have been reported in abstract form (Balice-Gordon and Lichtman, 1989, 1990a).

\section{Materials and Methods}

Neonatal mice were obtained from breeding colonies established in our animal care facility (CF1B strain initially obtained from Harlan/Sprague-Dawley, Inc.; C57B1 6J strain from Jackson Laboratories). The date a copulatory plug was found was designated embryonic day 0 (E0), and the date of birth was designated postnatal day $0(\mathrm{P} 0)$. Litters were culled to six pups on P4, and pups were weaned on P21. Female mice of the C57Bl 6J strain were found to be somewhat more tolerant of manipulation of their pups than CF1B females. Determination of sex after 2 weeks of age showed that approximately half of the animals used were male. No differences were noted between sexes or between strains of mice relevant to the present experiments.
Methods for anesthesia, preparation of animals, staining and imaging of neuromuscular junctions, and control procedures are described fully elsewhere (Lichtman et al., 1987; Balice-Gordon and Lichtman, 1990b). The intcrpretive limitations of thesc techniques are extensively discussed in Balice-Gordon and Lichtman (1991), and in this work we have attempted to surmount these by (1) making as many observations as possible, (2) extensive use of control experiments, and (3) using several different vital staining techniques. Only those methods specific to the present experiments are described below.

Visualization of motor nerve terminals and AChRs. Pups less than 1 week old were anesthetized by cooling $\left(\sim 10^{\circ} \mathrm{C}\right.$ for $\left.5-10 \mathrm{~min}\right)$ using a thermoelectric temperature-regulating device (a Peltier element driven by a Cambion Bipolar Controller) mounted in the microscope stage. Pups older than 1 week were anesthetized with chloral hydrate $(0.60$ $\mathrm{gm} / \mathrm{kg}$ ), delivered intraperitoneally with a 27 gauge needle, and were intubated and mechanically respirated (Harvard Instruments rodent ventilator).

Motor nerve terminals were stained with a $1 \mu \mathrm{M}$ solution of 4-Di-2ASP (Molecular Probes, Inc., Eugene, OR; Magrassi et al., 1987) perfused over the muscle for $2 \mathrm{~min}$. This low dose was used so that most of the dye could be washed away at the end of the experiment, enabling junctions to be restained 1-2 d later. Postsynaptic AChRs were usually labeled with a nonblocking dose of rhodamine-conjugated $\alpha$-bungarotoxin $(\mathrm{R} \alpha \mathrm{BTX})$ prepared according to the method of Ravdin and Axelrod (1977) so that synaptic transmission was only minimally affected ( $5 \mu \mathrm{g} / \mathrm{ml}$ for $3 \mathrm{~min}$; see also below). In some experiments, after obtaining images of 4-Di-2-ASP and R $\alpha$ BTX staining, motor nerve terminal membranes were stained with the voltage-sensitive dye RH795 (Molecular Probes, Eugene, OR; Grinvald et al., 1986) as described below. Neuromuscular junctions that were on the superficial surface of superficial muscle fibers were visualized using a $100 \times, 1.2 \mathrm{NA}$ water-immersion objective (Leitz NPL Fluotar), which was machined into a conical shape to fit more easily into the neck of a neonatal mouse. Images were obtained using a silicon-intensified target (SIT) video camera (series 66, Dage-MTI, Inc.) and an image processor (Trapix, Recognition Concepts, Inc.) as described previously (Lichtman et al., 1987; Balice-Gordon and Lichtman, 1990b). Axon branches were difficult to image in a single focal plane because they often traveled vertically from nerve branches deep within the muscle. Nonetheless, for each junction studied, as many images were obtained as necessary to determine unambiguously the number and position of the innervating axons. Junctions in which the number of innervating axons could not be determined were not studied further. Because of the curvature of individual muscle fibers and the orientation of the muscle in the mouse, areas within a junction were often in different focal planes. Thus, in some figures, portions of images of different focal planes have been spliced together using the image processor and interactive software. Alignment between motor nerve terminals and underlying acetylcholine receptors was determined after 4-Di-2-ASP staining using images of 4-Di-2-ASP "bleedthrough" into rhodamine filters as previously described (BaliceGordon and Lichtman, 1990b).

Once images of selected neuromuscular junctions were obtained, the wound was closed with $8-0$ silk and painted with a liquid bandage preparation (NewSkin, MedTech Lab., Inc.). Pups were warmed to room temperature using a temperature-controlled blanket (Harvard Instruments) or an infrared heat lamp. When pups became alert and responsive (cold-anesthetized pups, 5-30 min later; chloral hydrate-anesthetized pups, 6-8 hr later), they were returned to their mothers. Pups requiring a longer time to recover fully from the anesthetic were kept warm and fed drops of milk at $2 \mathrm{hr}$ intervals. Although female mice frequently cannibalized manipulated pups, about $60 \%$ survived until the next experiment.

One to seven days later, the staining and visualization procedures were repeated. The neuromuscular junctions stained at the first view were found by scanning the muscle surface with a $50 \times, 1.0 \mathrm{NA}$ waterimmersion fluorescence objective (Leitz) while referring to the pictures taken in the initial experiment. Observations of neuromuscular junctions were repeated at least once and as often as seven times during the first 3 postnatal weeks.

Determination of relative density and turnover of AChRs in postsynaptic areas. To compare the relative density of AChRs in faint and normally staining areas of junctions, the output of the SIT video camera was calibrated (Rich and Lichtman, 1989a). We adjusted the gain settings of the video camera and the intensity of the incident light used so that the camera was operating in its linear range when viewing $\mathrm{R} \alpha \mathrm{BTX}$ staining of postsynaptic AChRs as measured by the image processor. 
In order to measure the value of the camcra gain and offsct, a calibration curve was generated by measuring the brightness of the same R $\alpha$ BTXstained postsynaptic AChR areas with a series of neutral-density filters of known transmittance (Rolyn Optics) placed in the incident light path. We then asked how much filtration of the bright areas was necessary to obtain a brightness reading equivalent to that of a faintly stained area within the same junction measured with no filtration. Because these measurements were made in the linear range of the SIT camera signal and the slope (gain) and y-intercept (offset) were measured, this value is an accurate estimate of the relative number of R $\alpha$ BTX-stained AChRs in faintly stained postsynaptic areas.

To determine how AChR areas were lost from junctions, receptors were labeled with a saturating dose of $\operatorname{R} \alpha \mathrm{BTX}(5 \mu \mathrm{g} / \mathrm{ml}$ for $1 \mathrm{hr})$ and their intensity recorded digitally as described above. At the second view, typically $6-48 \mathrm{hr}$ later, the remaining original staining with $\mathrm{R} \alpha \mathrm{BTX}$ was reimaged to examine the distribution of $\mathrm{AChRs}$ remaining since the first view. Additional $\mathrm{R} \alpha \mathrm{BTX}$ was then applied to label new AChRs inserted in the junctions since the first observation and the junctions were digitally photographed again. The area of faintly staining AChR regions or of $A C h R$ regions that were lost between views was measured using a digital bit pad (Summagraphics).

$V$ ital labeling of motor nerve terminal and axon membranes. Because 4-Di-2-ASP labels only intracellular organelles such as mitochondria (Lichtman et al., 1989), we also screened several different classes of fluorescent dyes for their ability to vitally stain motor nerve terminal membranes. Although several different fluorescent compounds were found that labeled the membranes of axons and nerve terminals without damaging them (R. J. Balice-Gordon and J. W. Lichtman, unpublished observations), voltage-sensitive dicationic styryl pyridinium dyes developed by Grinvald and his colleagues (see Grinvald, 1985, for review) such as RH795 and RH414 resulted in bright nerve terminal membrane staining with low background fluorescence.

To visualize motor nerve terminal membranes, junctions were stained for 3-5 min with a $50 \mu \mathrm{M}$ solution of RH795 (Molecular Probes, Eugene, OR; Grinvald et al., 1986) diluted in sterile Ringer's (Travenol) and filtered through a $0.2-\mu \mathrm{m}$-pore filter. RH414 was used in some experiments with similar results. Although these dyes can be used as activitydependent labels (Betz and Bewick, 1992), motor axons were not stimulated in the present experiments. Following staining, excess dye was washed away with Ringer's for 10-30 min; this washing also diminished nonspecific background staining. Junctions were then visualized with conventional fluorescein optics.

Motor axon staining was typically more intense than nerve terminal staining with these compounds, especially when axons were myelinated. Blood vessels, connective tissue cells, and perisynaptic cells were more weakly labeled. As with other dyes, to prevent fading or photodamage to the tissue, the output of a $100 \mathrm{~W} \mathrm{Hg}$ lamp was attenuated at least $70 \%$ with neutral-density filters. RH795 staining had no apparent shortor long-term toxic effects on junctions (see Results). However, concentrations in excess of $100 \mu \mathrm{M}$ were sufficient to occasionally cause spontaneous contractions of muscle fibers, vasoconstriction (London et al., 1989), and damage to neuromuscular junctions upon illumination. Ideally, developing junctions would have been stained with all three markers (RH795, 4-Di-2-ASP, and R $\alpha$ BTX) and followed over time. This proved difficult because, while RH795 staining was excellent at the first view, at a second view 1-2 d later the accumulated background staining from RH795 obscured staining of the nerve terminal. At longer intervals (weeks to months), however, the background staining diminished so that restaining was possible (e.g., see Fig. 16).

\section{Results}

The results are divided into seven sections. The first section describes a correlation between multiple innervation and the distribution of postsynaptic AChRs. Sections 2 and 3 describe AChR depletion from areas of the postsynaptic membrane within junctions. Sections 4-6 describe the spatial and temporal relationship between nerve terminal elimination and AChR loss. The final section describes the fate of neuromuscular junctions once the period of synapse elimination is complete.

\section{AChR distribution differs at singly and multiply innervated junctions}

4-Di-2-ASP staining (as well as more conventional histological (see Fig. 1 caption; Steinbach, 1981) and physiological techniques (J. Nabekura, H. Colman, and J. W. Lichtman, unpublished observations) showed that in the mouse sternomastoid muscle, the transition from multiple to single innervation takes place during the first 2 postnatal weeks. Shortly before birth, more than $90 \%$ of the neuromuscular junctions in the sternomastoid muscle have at least two different motor axons innervating the junction (Fig. 1). In a few junctions $(<5 \%)$, three different motor axons could be seen innervating the junction. Seven days after birth, however, only approximately $40 \%$ of the junctions are innervated by two motor axons; the remainder are singly innervated. By the end of the second postnatal week, virtually none of the muscle fibers are innervated by more than one axon.

As has been observed by other workers in other animals and in other muscles (Steinbach, 1981; Slater, 1982a), at birth (when nearly all of the muscle fibers are multiply innervated) AChRs are arranged into bands or branches interposed with areas (when muscle fibers are uniformly singly innervated) AChRs are arranged into bands or branches interposed between areas of very low receptor density. To determine whether this change in AChR distribution correlates with the transition from multiple to single innervation at individual junctions, rather than being a fortuitous correlation of two independent events that are both a function of animal age, we studied receptor staining in individual junctions from 6-7-d-old mice in which approximately half of the muscle fibers were multiply innervated. As can be seen in Figure 2, at this age 4-Di-2-ASP staining shows some junctions receive input from two axons whereas others receive input from a single axon. Junctions that were multiply innervated on P6-P7 were more plaque-like (having a relatively small percentage of junctional area with very low receptor density; Fig. 2, junction 3) than junctions that were singly innervated at the same age, which were more broken up (having a higher percentage of junctional area with very low receptor density; Fig. 2, junction 2). Of approximately 100 junctions from two 7-d-old mice studied in this way, 18 were multiply innervated, 15 were singly innervated, and the rest either were not well positioned (i.e., on their sides; see, e.g., Fig. 2, junction 1) or their axons were oriented in such a way that it was difficult to determine whether they were multiply or singly innervated. The percentage of junctional area that had very low or undetectable levels of AChRs was only $13.3 \pm 9.9 \%$ in multiply innervated junctions compared to $29.7 \pm 14.1 \%$ in singly innervated junctions (Student's $t$ test, significant at $p<0.001$ ). Thus, individual neuromuscular junctions must undergo a change in AChR distribution from plaque to branched forms and synapse elimination at roughly the same time.

\section{Postsynaptic areas undergoing AChR loss are commonly seen in developing neuromuscular junctions}

To understand how areas of very low AChR density appear in junctions undergoing the transition from plaque to branched forms, we stained junctions with a nonblocking dose of $\mathrm{R} \alpha \mathrm{BTX}$ to label AChRs and then reviewed this staining at $2 \mathrm{~d}$ intervals. On P14 and P21, additional R $\alpha$ BTX was applied and the junctions viewed again $(N=58$ junctions from 14 P6-P8-d-old mice; 19 junctions viewed three times, 21 viewed four times, 7 viewed five times, 10 viewed six times, and 1 viewed seven times).

As might be expected, junctions that appeared relatively broken up into branches at the initial view showed fewer changes 


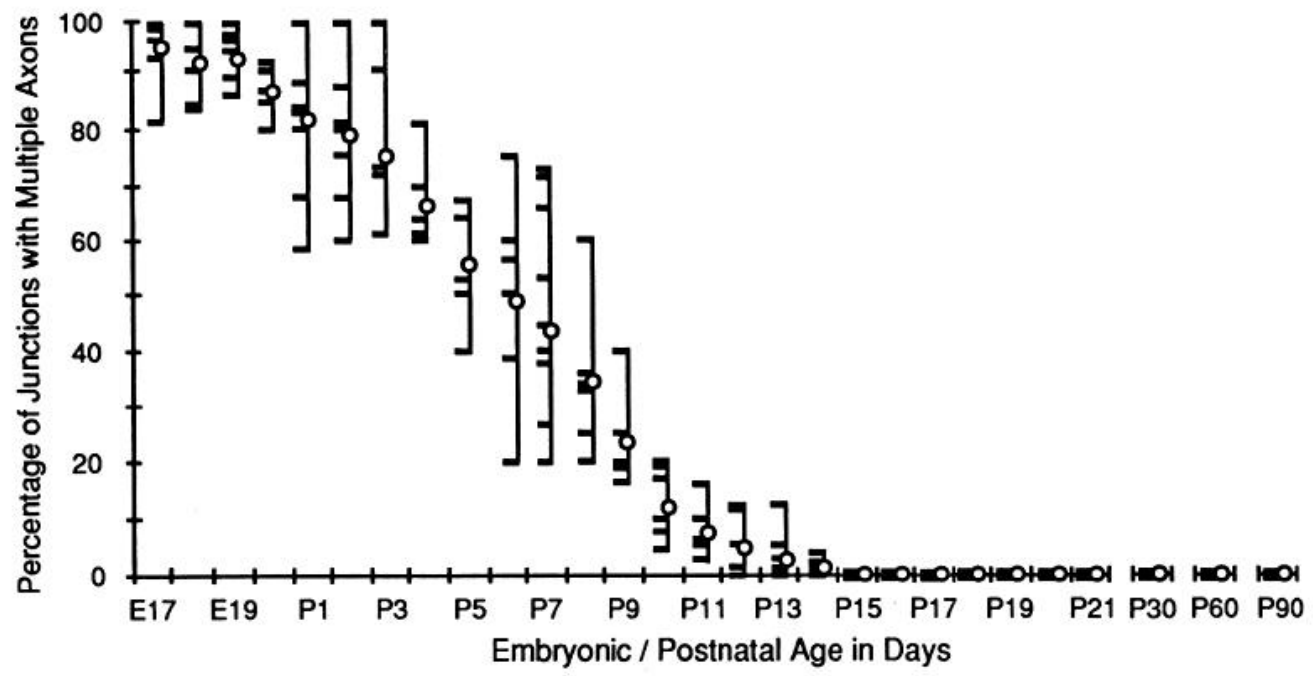

Figure 1. Most neuromuscular junctions in the mouse sternomastoid muscle are innervated by a single motor axon by 2 weeks of age. Junctions were stained with 4-Di-2-ASP in mice ranging in age from E17 to P90. Each horizontal line represents the percentage of junctions innervated by more than one axon determined by examining between 17 and 61 junctions in each of five to nine animals at each age. Open circles represent mean values. We do not know whether the range of data at any one age represents variation in the rate of synapse elimination between muscles or the relatively small sample sizes that gave rise to each individual value. Other anatomical techniques such as tetranitroblue tetrazolium (TNBT) staining (Jordan et al., 1988) or combined silver/AChE staining (Oda, 1986) in fixed whole-mount preparations showed a similar incidence of multiple innervation at each age examined (for each type of staining, at least 25 junctions were examined from each of five muscles at each age). For example, 4-Di-2-ASP staining showed that $42 \pm 21 \%$ of sternomastoid neuromuscular junctions remained multiply innervated on P7, while TNBT or silver/ AChE staining showed that $44 \pm 13 \%$ and $47 \pm 9 \%$ of junctions, respectively, were multiply innervated at this age (values not significantly different, Student's $t$ test). These observations showed that the incidence of multiple innervation can be as reliably determined using vital staining in situ as it can be with conventional staining of fixed tissues removed from the animal.
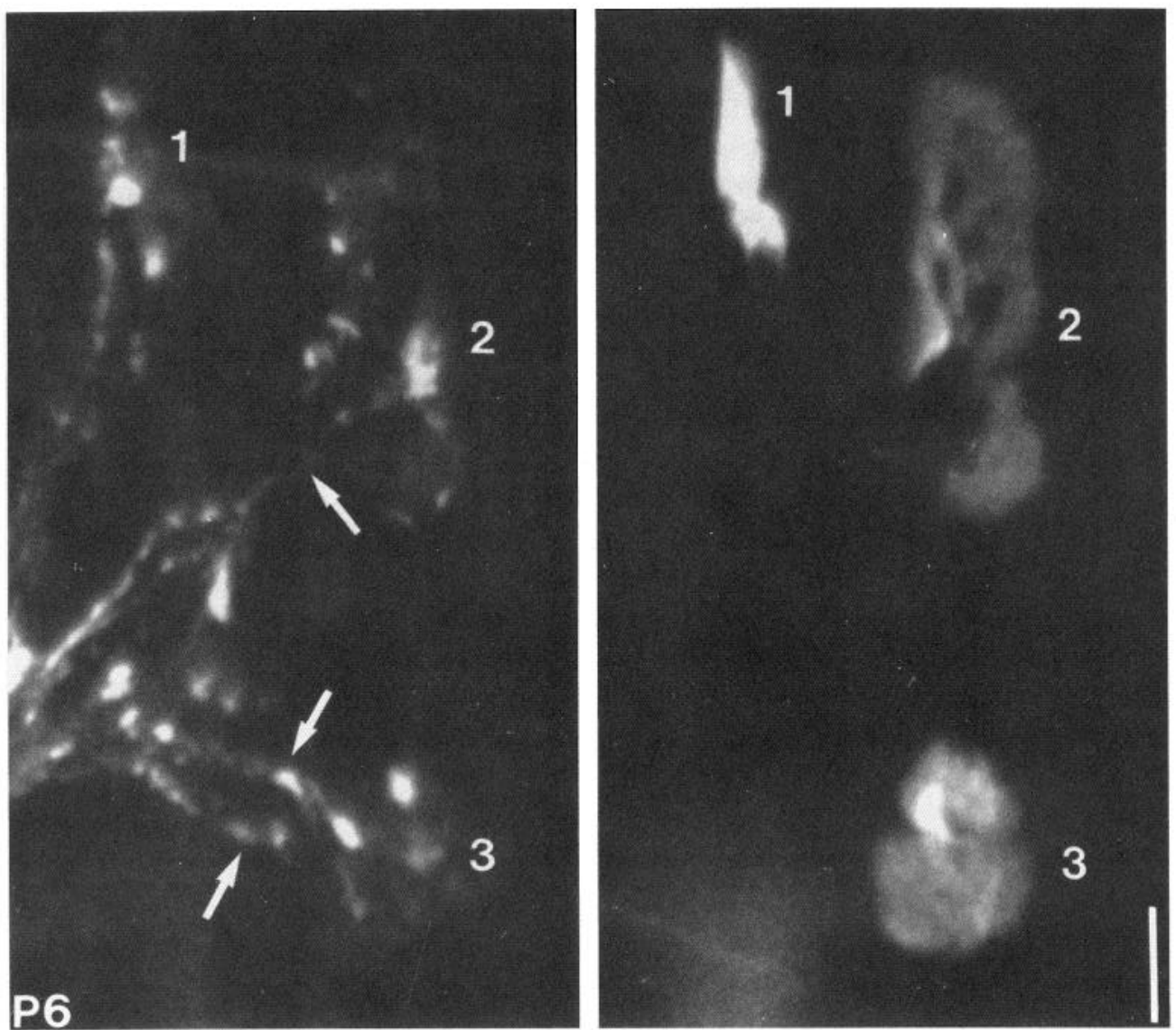

Figure 2. AChR distribution differs at multiply and singly innervated junctions. Shown are three junctions $(1-3)$ in a sternomastoid muscle from a 6-dold mouse stained with 4-Di-2-ASP (left panel) and R $\alpha \mathrm{BTX}$ (right panel). Junction 2 is innervated by one motor axon (arrow), and the AChR distribution at this junction is relatively broken up in appearance, having areas of high AChR density (bright regions) interspersed with areas of relatively low AChR density (dark regions). In contrast, junction 3 is innervated by two motor axons (arrows) and its AChR distribution is relatively homogeneous and plaque-like. Junction 1 is also multiply innervated, but its AChR distribution was only partially visible due to the curvature of the muscle fiber. Such junctions were not studied further. Scale bar, $20 \mu \mathrm{m}$. 

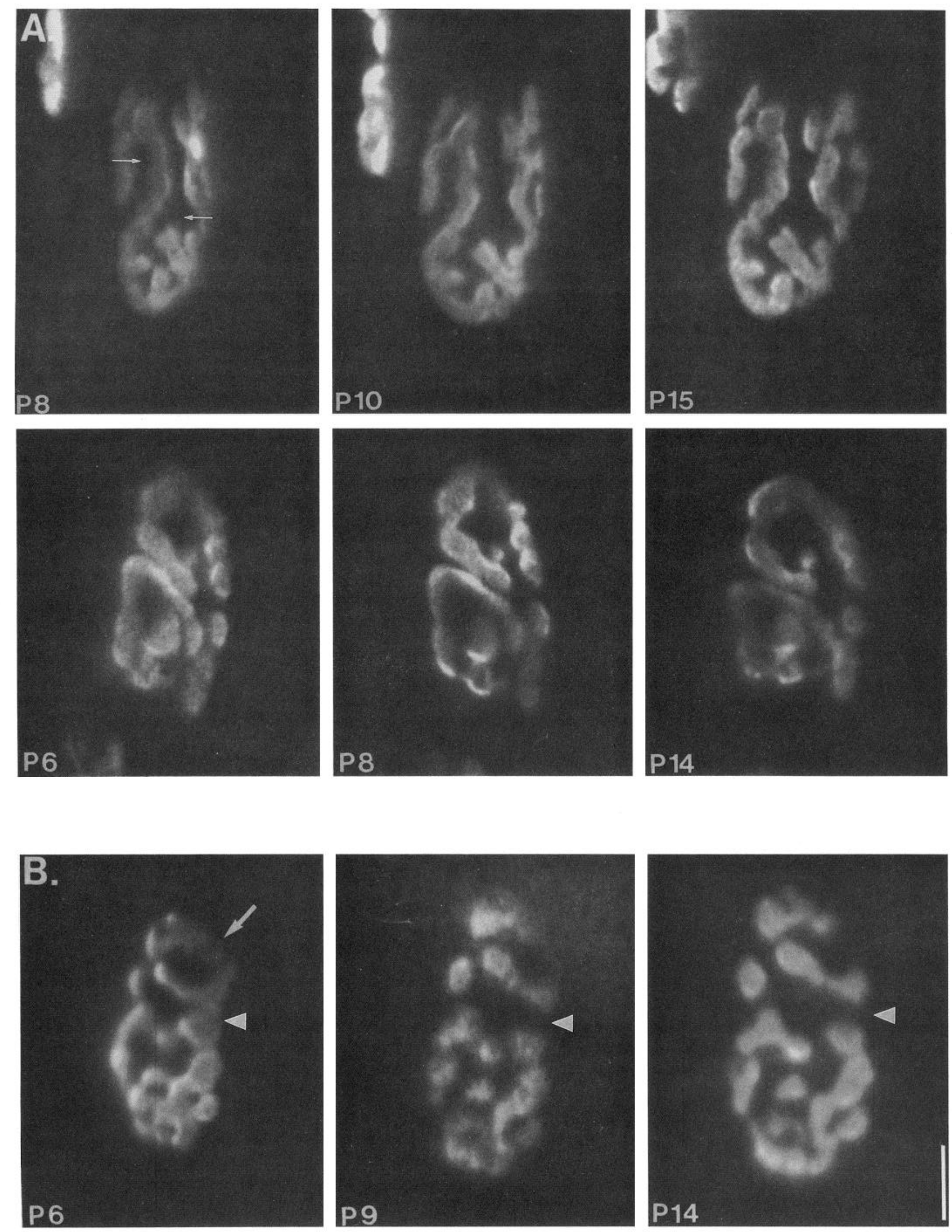
in $\mathrm{AChR}$ distribution than junctions that appeared more plaquelike. Indeed, in 19 junctions that were already broken up at the first view, with more than one-third of the junctional area consisting of very low AChR density, no loss of AChR-rich areas was observed (Fig. $3 A$ ). We presume that such junctions underwent loss several days earlier, because nearly all junctions at P0 (>90\%) had a plaque-like AChR distribution (see section 4 below; Steinbach, 1981; Slater, 1982a). A few junctions (3 of 58) that appeared to have a moderate degree of branching at the first view lost one or two small AChR-rich areas between P8 and P14 (Fig. 3B).

In the remaining junctions we studied (36 of 58), AChRs were distributed into more homogeneous plaques at the first view. In each of these junctions, multiple discrete postsynaptic areas stained with $\mathrm{R} \alpha \mathrm{BTX}$ on P6 were no longer visible at subsequent views on $P 8, P 10$, and $P 12$ (Fig. 4). During this time, junctions that showed evidence of loss of previously inserted AChRs from postsynaptic areas lost a mean of $4.3 \pm 0.5$ areas each. Typically, these areas were relatively small, ranging in size from 6 to 58 $\mu \mathrm{m}^{2}$ (mean, $24 \pm 10 \mu \mathrm{m}^{2} ; N=167$ areas, 39 junctions) compared to a mean junctional area of $319 \pm 43 \mu \mathrm{m}^{2}$ on P6-P8 $(N=58$ junctions).

Because the views from P8 through P12 were made without adding additional $\mathrm{R} \alpha \mathrm{BTX}$, these results show that previously inserted AChRs in the postsynaptic membrane are lost from several areas within individual junctions. This loss could mean either that AChRs in these areas undergo more rapid turnover than receptors elsewhere in the junction, in which case insertion of receptors into these areas may also be increased, leading to no net change in receptor density; or that these areas may undergo a net loss of AChRs if the loss of previously inserted receptors is not compensated for by an increased rate of insertion of new receptors into those areas. The junctions were thus restained with R $\alpha$ BTX on P14 and again on P21 to determine the receptor density in these areas.

Every one of the areas which lost AChR staining between P6 and P12 (167 areas in 39 junctions) could not be restained on P14 or P21 (Fig. 3B, panel P14; Fig. 4, columns P14, P21). Furthermore, between P12 and P14, a few additional AChRrich areas were lost. The inability to stain AChRs in postsynaptic areas that lost previously inserted receptors shows that the areas which disappear undergo a net depletion of AChRs, due to loss of previously inserted receptors that are not replaced by newly inserted receptors.

Examination of successive views of each junction showed that postsynaptic areas within a junction were depleted of AChRs sequentially rather than simultaneously (Fig. 4). For example, in the junction illustrated in the first row of Figure 4, two small AChR-rich areas present on P6 were depleted of receptors by P8. Another area present on P10 was depleted of receptors by P12. One additional area was depleted of AChRs between P12 and P14. In most of these junctions, periods of depletion of
AChR-rich areas were interspersed with periods of relative quiescence, indicating a discontinuous process. In the junction illustrated in the first row of Figure 4, no postsynaptic areas were depleted of AChRs between P8 and P10.

Despite the loss of multiple AChR-rich areas within a junction, there was no net decrease in their total AChR-rich area. This came about because during the first 2 postnatal weeks while AChR-rich areas were being lost, there was substantial growth of the junction as a whole. In the example illustrated in the first row of Figure 4, five AChR-rich areas totaling $128 \mu \mathrm{m}^{2}$ were lost between $\mathrm{P} 6$ and $\mathrm{P} 14$ but the total area of the remaining AChR regions increased from $203 \mu \mathrm{m}^{2}$ on P6 to $315 \mu \mathrm{m}^{2}$ on P14. A comparable increase in area was seen in every junction viewed more than once between P6 and P14.

However, in none of the junctions we followed during the first weeks after birth was the steady increase in synaptic area accounted for by the significant addition of new AChR-rich areas that changed the shape of the junctions. Rather, synaptic area increased by an overall enlargement of the existing postsynaptic territory: not only did AChR-rich areas enlarge, but the spaces between AChR-rich areas also enlarged. The ongoing expansion of synaptic and intrasynaptic areas within the junction during the first 2 postnatal weeks is analogous to the mode of intercalary enlargement of junctions in older animals (BaliceGordon and Lichtman, 1990b). Thus, the loss of AChR-rich areas is not compensated for by a comparable addition of discrete new areas.

One consequence of this form of generalized enlargement was the appearance of obvious gaps in what formerly appeared to be contiguous AChR-rich areas when the junction was more compact (e.g., see Fig. $3 B$, region marked with arrowheads; see also Fig. 11). The appearance of gaps as well as the depletion of AChRs from postsynaptic areas described above contributed to the breaking apart of initially plaque-like receptor distributions. The areas that were depleted of AChRs, however, were usually easy to distinguish from the gaps that appeared due to growth. In many of the areas that we judged to have been depleted of AChRs, some element in the postsynaptic receptor pattern had disappeared rather than been displaced (see Fig. 11). Another difference between $\Lambda \mathrm{ChR}$ areas that were lost and the gaps that appeared was that intermediate views sometimes caught the regions that would ultimately be completely depleted of receptors at a time when they stained more faintly with $\mathrm{R} \alpha \mathrm{BTX}$ (Fig. 4, first row, small arrows; see also Fig. 5).

In all but one of the junctions we viewed another time after 2 weeks of age, the postsynaptic AChR-rich area remained stable, undergoing no further losses. In the one junction which did change (illustrated in the last row of Fig. 4), one small area (9 $\mu \mathrm{m}^{2}$ ) was lost between 14 and $21 \mathrm{~d}$ of age. Loss of AChR-rich areas only rarely occurs in sternomastoid neuromuscular junctions in normal adult animals (see section 7 below; Balice-Gordon and Lichtman, 1990b, 1991). On the other hand, loss of

Figure 3. Junctions with AChR distributions that appeared relatively branched at the first view show little or no loss of receptor areas at subsequent views. Shown are three junctions stained with $\mathrm{R} \alpha \mathrm{BTX}$ at the first view (left panels) that were restained and rephotographed on two subsequent occasions (middle and right panels). A, More than $30 \%$ of the area of these two junctions (top and middle rows) consists of areas of very low or undetectable AChR density (dark regions; see, e.g. top left panel, arrows). Comparison of AChR-rich areas in each view shows that junctions such as these that were already broken up at the first view often did not show evidence of loss of AChR-rich regions at subsequent views. $B$, Shown is a junction that is relatively branched at the first view. Subsequent views show that AChRs have been depleted from the upper right region of the junction (arrow). The remaining regions have spread apart during this time, and gaps that were present in the original pattern have become more evident (arrowheads). Scale bar, $20 \mu \mathrm{m}$. 

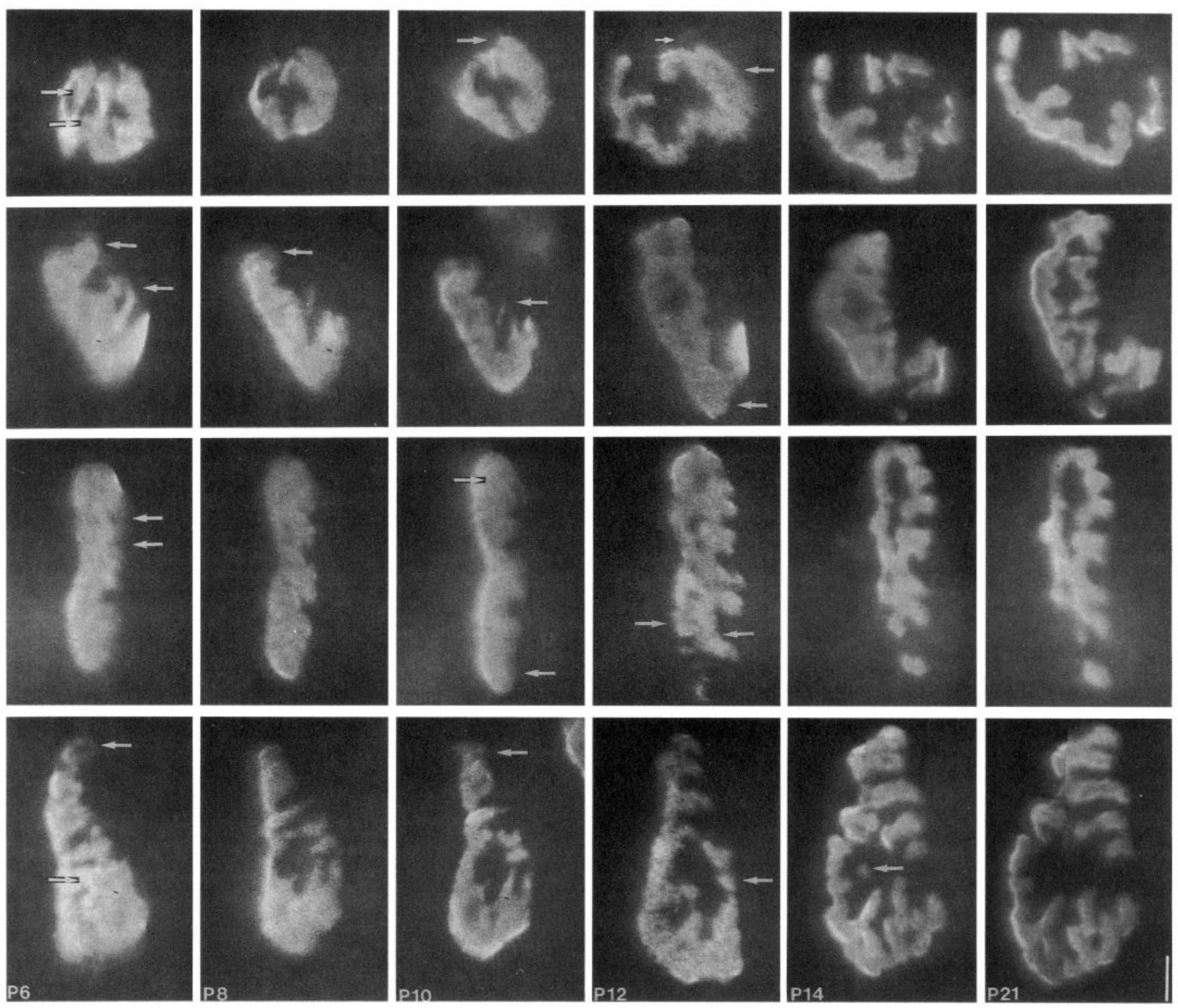

Figure 4. Junctions with relatively plaque-like AChR distributions lose multiple AChR-rich areas in succession during the first 2 postnatal weeks. The first panel in each row illustrates a neuromuscular junction in a sternomastoid muscle from a P6 mouse in which postsynaptic AChRs were stained with $\mathrm{R} \alpha \mathrm{BTX}$. Adjacent panels show the same junction rephotographed on P8, P10, and P12 without restaining. It was necessary to restain junctions with R $\alpha$ BTX on P14 and P21 because turnover of existing AChRs resulted in junctional staining being too dim to image. Thus, junctions were restained with $\mathrm{R} \alpha \mathrm{BTX}$ and rephotographed on P14 and again on P21. In each row, comparison of adjacent panels shows that small AChRrich areas within the junction disappear one after the other (arrows). In the junction in the first row, one AChR area present on PIO (arrow) appears fainter on P12 (small arrow) and is no longer present on P14. In most junctions, loss of AChR-rich areas ceased by 2 weeks after birth. In the junction illustrated in the bottom row, one small spot of staining was lost between P14 (arrow) and P21. Note that the principal means of junctional growth is the expansion of remaining AChR-rich areas and the spaces between them. Scale bar, $10 \mu \mathrm{m}$.

AChR-rich areas is commonly observed in adult neuromuscular junctions undergoing synapse elimination following reinnervation (Rich and Lichtman, 1989a).

\section{Depletion of AChRs from each postsynaptic area is gradual}

To determine how AChRs are depleted from a postsynaptic area, receptors already inserted in the muscle fiber membrane were labeled with $\mathrm{R} \alpha \mathrm{BTX}$ at one view and the staining intensity was measured at later times $(N=258$ junctions, 39 mice; mean age at first view, $7 \pm 0.5 \mathrm{~d}$; at second view, $1-4 \mathrm{~d}$ later, mean, $3 \pm 0.8 \mathrm{~d}$ ). As with some of the junctions from 1-week-old mice that were viewed three or more times described in section 2 , in this series of experiments, in which junctions from 1-week-old mice were viewed twice, about half (134 of 258) showed no evidence of loss of AChR-rich areas. These junctions are likely to have undergone loss at an earlier time (see also section 4 below).

In some of these junctions (104 of 258), AChR-rich areas that stained as brightly as the rest of the junction at the first view were no longer present at the second view several days later (Fig. 5A). In some junctions $(N=20)$, we observed faintly stained $\mathrm{AChR}$ areas at the first view (Fig. $5 A$, lower arrow; Fig. $5 B$ ). These faintly stained areas were completely depleted of AChRs at the second view. Because postsynaptic areas that stained faintly with $\mathrm{R} \alpha \mathrm{BTX}$ were found in junctions the first time that they 

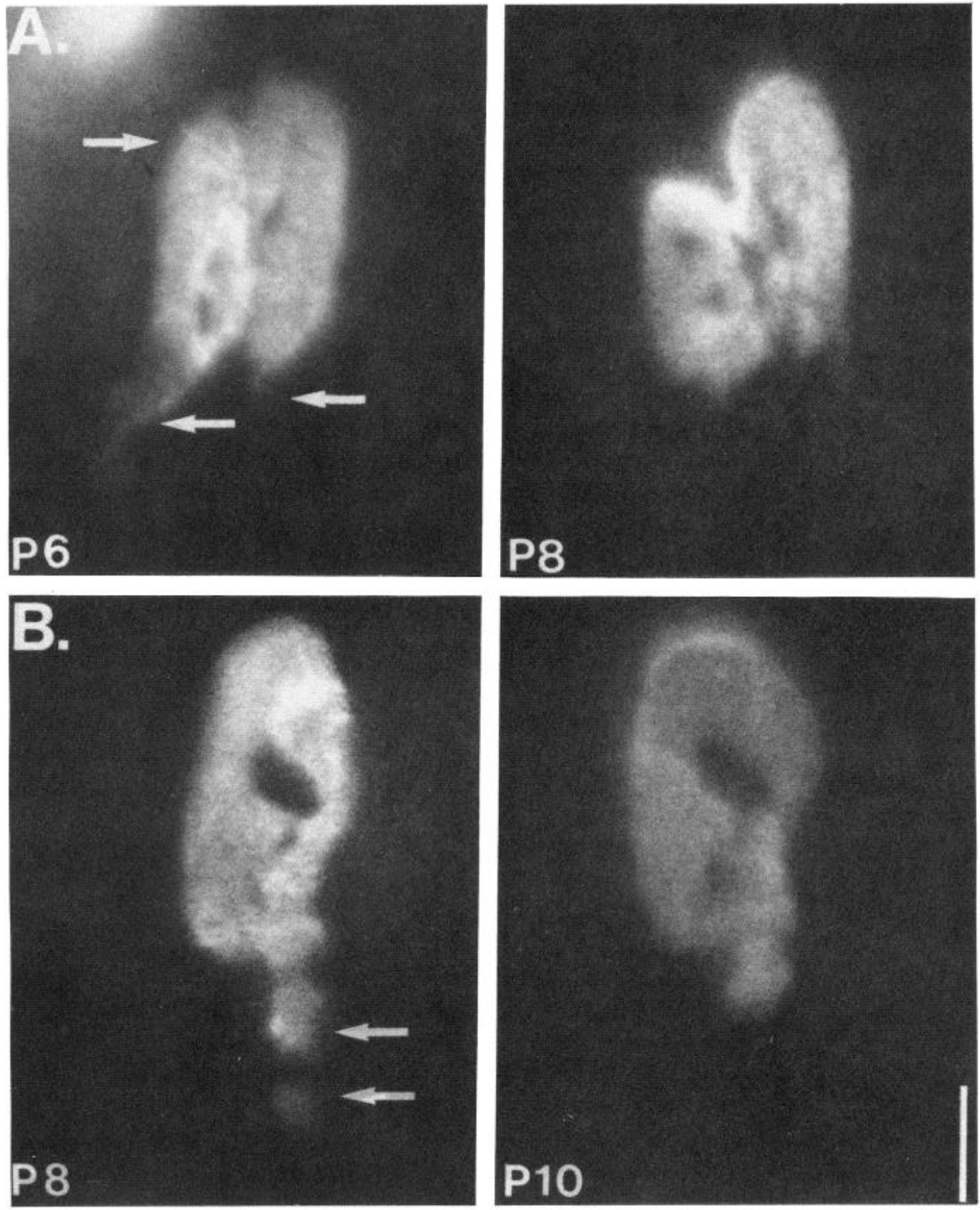

Figure 5. Postsynaptic areas are gradually depleted of previously inserted AChRs. Two junctions are shown in the process of losing AChR-rich areas. In the left panels, the junction has been stained with $\mathrm{R} \alpha \mathrm{BTX}$. The right panels show the original $\mathrm{R} \alpha \mathrm{BTX}$ staining rephotographed $2 \mathrm{~d}$ later. $A$, In this junction, postsynaptic AChR-rich areas that on P6 stained more faintly than the rest of the junction (lower arrows) were completely depleted of previously inserted AChRs by P8. Other areas (top arrow) may also have been depleted of previously inserted AChRs in the interval between views. $B$, In this junction, a postsynaptic $A C h R$-rich area that on $P 8$ stained more faintly than the rest of the junction (lower arrow) was completely depleted of previously inserted AChRs by $P 10$. Another area that on $P 8$ stained as brightly as the rest of the junction (upper arrow) seems to have been partially displaced upward in the interval between views. This type of change may mean that $\mathrm{AChRs}$ are migrating in the plane of the muscle fiber membrane (see Fig. 7). Scale bar, 10 $\mu \mathrm{m}$. were viewed, it is unlikely that loss of AChRs was induced by labeling receptors with $\mathrm{R} \alpha \mathrm{BTX}$ or by other aspects of the viewing procedure. By $14 \mathrm{~d}$ of age, faintly stained $\mathrm{AChR}$ areas were very rare (Fig. 6), as was evidence of loss of AChR-rich areas. Thus, the data suggest that all faintly stained receptor areas are in the midst of being depleted of AChRs.

We retrospectively compared the brightness of AChR-rich areas that 2-3 d later (mean, $2 \pm 0.3 \mathrm{~d}$ ) were observed to disappear to the receptor-rich areas in junctions that remained intact ( $N=49$ junctions, $8 \mathrm{P} 7$ mice, 71 areas). Not surprisingly, these brightnesses varied widely. In order to obtain a quantitative approximation of the relative numbers of AChRs in faintly stained compared to brightly stained postsynaptic areas, the output of the SIT camera was calibrated as described in Materials and Methods. Postsynaptic areas stained with $\mathrm{R} \alpha \mathrm{BTX}$ which are still easily discernable but appear faint were estimated to contain between $31 \%$ and $86 \%$ as much $\mathrm{R} \alpha \mathrm{BTX}$ staining as parts of the junction which are brightly stained (mean, $58 \pm 12$ $\% ; N=10$ junctions from three P7 mice). The wide range of values suggests that the density of AChRs in areas undergoing receptor depletion changes from the amount initially present in the rest of the junction to ultimately a very low density indistinguishable from background.
To determine how rapidly AChRs are lost from individual postsynaptic areas, muscles were stained with $\mathrm{R} \alpha \mathrm{BTX}$ and plaque-like junctions were monitored over the course of $1 \mathrm{~d}(\mathrm{~N}$ $=31$ junctions, 23 P6-P8 mice). In 16 junctions, changes in the brightness of AChR staining were discernable less than $24 \mathrm{hr}$ after the first view (Fig. 7A). In four junctions, faint $\mathrm{AChR}$ areas were viewed a second time just $6 \mathrm{hr}$ after the first view was made. In each case, a significant loss of staining intensity was discerned.

In some junctions $(N=11)$, a portion of the labeled AChRs in areas undergoing loss appeared to be redistributed in the postsynaptic membrane in the brief interval between views. In the junction shown in Figure 7, at the time AChRs were found to have been depleted from the upper portion of the junction, the distribution of labeled receptors had also changed. In particular, postsynaptic regions that did not contain $\mathrm{R} \alpha \mathrm{BTX}$ staining at the first view (Fig. $7 B$, left panel, arrows) did contain some $\mathrm{R} \alpha \mathrm{BTX}$ staining $17 \mathrm{hr}$ later (Fig. $7 \mathrm{~B}$, right panel, arrows). Application of additional $\mathrm{R} \alpha \mathrm{BTX}$ at the final view showed a similar pattern of loss and changes in overall receptor density and distribution; thus, $\mathrm{R} \alpha \mathrm{BTX}$-labeled AChRs are unlikely to have behaved differently from unlabeled receptors in the same junctions. These observations indicate that depletion of AChRs 
Figure 6. AChR areas within junctions that stain faintly with $\mathrm{R} \alpha \mathrm{BTX}$ are commonly observed during the first 2 postnatal weeks and are rare after $14 \mathrm{~d}$ of age. The percentage of junctions containing faint $\mathrm{AChR}$ areas was determined by examining at least 10 junctions in each of five muscles stained with $\mathrm{R} \alpha \mathrm{BTX}$ at each age. Faint AChR areas are approximately $30-90 \%$ as bright as the rest of the receptor-rich areas and are typically greater than 5 $\mu \mathrm{m}^{2}$ in size. See Results and Materials and Methods for details. At the time multiple innervation in the muscle is declining the most rapidly (see Fig. 1), faint AChR areas are the most prevalent. Bars indicate average values and lines indicate SD.

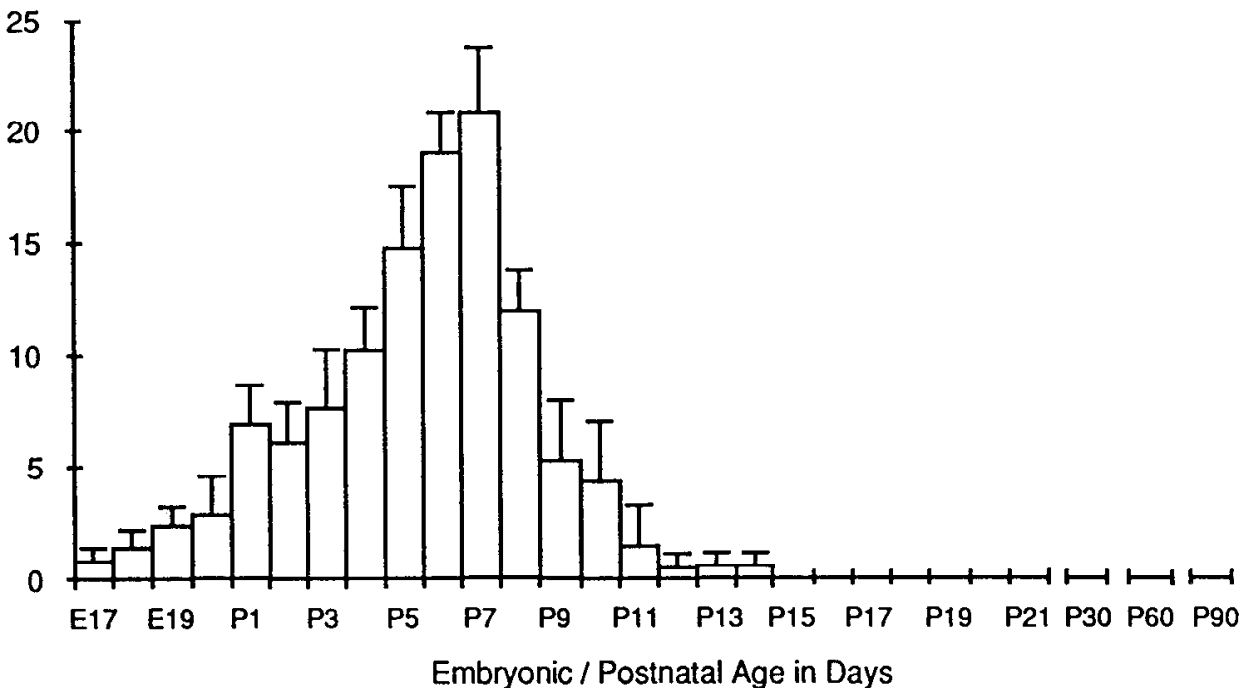

from postsynaptic membrane areas can be rapid. In each case studied, once postsynaptic areas began to lose AChRs, the remaining receptors in those areas invariably disappeared completely in less than $24 \mathrm{hr}$.

\section{Multiply innervated junctions are more likely than singly innervated junctions to lose $A C h R$-rich areas}

Given that multiply innervated junctions are typically plaquelike and that singly innervated junctions arc more branched (see section 1 above), loss of AChR-rich areas may occur during (and perhaps because of) the transition from multiple to single innervation. We thus wanted to study the $\mathrm{AChR}$ distribution at individual junctions that we knew were undergoing synapse elimination. As a first step, we observed junctions stained with 4-Di-2-ASP multiple times to ensure that this dye could be used to follow changes in junctional innervation over time in living neonatal mice. Junctions from 4-8-d-old mice (mean age, $6 \pm$ $0.4 \mathrm{~d})$ were stained with 4-Di-2-ASP, and the number of innervating axons was determined ( $N=360$ junctions, 74 mice). Junctions were then restained and relocated 1-4 d later (mean, $3 \pm 0.3 \mathrm{~d}$ later; 291 junctions viewed twice and 32 junctions viewed three times). An additional 37 junctions were unable to be studied at the second view because their muscle fibers had shifted, altering the orientation of junctions onto their side, which in these cascs partially obscured either the axons or the synaptic sites.

In 148 of 360 junctions, 4-Di-2-ASP staining showed two motor axons entering the junctional area at the first view (Fig. 8 , left panels). In 116 of these 148 junctions, 4-Di-2-ASP staining at the second view showed that only one axon remained (Fig. 8, right panels). In some cases, a second axon was still present at the second view but no longer projected all the way to the junction (Fig. 8, bottom row). In 32 of 148 junctions, two motor axons were still present at the second view (see, e.g., Fig. 10). However, in each of these junctions, only one axon projected to the junction when a third view was made $1-4 \mathrm{~d}$ later (mean, $2 \pm 0.1 \mathrm{~d}$ ). In the junctions which were singly innervated at the first view ( 175 of 360 ), there was no evidence at the second view either that the single axon withdrew from the junction or that a second axon appeared.

We compared a group of junctions that several days previously had been stained and viewed with junctions from littermate control animals that had never been previously stained and viewed. No significant differences in junction size, extent of multiple innervation, or time course of synapse elimination were observed (comparison by age or weight). For example, 7 $\mathrm{d}$ after birth, $41 \pm 6 \%$ of junctions in the control group were innervated by two motor axons ( $N=162$ junctions, 18 mice). In a group of mice whose junctions were stained and viewed twice (first view on $\mathrm{P} 4$ and second view on $\mathrm{P} 7$ ), $43 \pm 4 \%$ were multiply innervated on $\mathrm{P} 7$ ( $N=50$ junctions, 7 mice). Thus, axon withdrawal appeared to be unaffected by the staining and viewing procedures we used.

To correlate the depletion of AChRs from postsynaptic areas directly with the transition from multiple to single innervation, junctions were stained both with 4-Di-2-ASP (to assess the number of innervating motor axons) and with R $\alpha \mathrm{BTX}$ and were then viewed repeatedly ( $N=376$ junctions, 74 P4-P8 mice; mean age at first view, $5 \pm 0.6 \mathrm{~d} ; 312$ junctions viewed twice and 64 junctions viewed three times). 4-Di-2-ASP staining showed that 257 junctions were innervated by two motor axons and 119 were innervated by one motor axon at the first view. We found

Figure 7. Postsynaptic areas are rapidly depleted of previously inserted AChRs. A, Shown is a junction from a P6 mouse (left panel) stained with RaBTX. The right panel shows the same junction $17 \mathrm{hr}$ later. No additional $\mathrm{R} \alpha \mathrm{BTX}$ had been applied; thus, the labeled AChRs are those that have remained in the junction since the first view. The top one-third of the junction has been depleted of AChRs in this brief interval and thus appears faint. $B$, The top one-third of the junction is shown at higher magnification. A portion of the labeled AChRs in this region appear to have been redistributed in the postsynaptic membrane. Three regions that did not contain R $\alpha$ BTX staining at the first view did contain some staining $17 \mathrm{hr}$ later (compare regions marked with arrows in left and right panels). This comparison was made by taking the two images with identical illumination and gain settings of the SIT video camera. For illustration purposes only, the right panels have been enhanced so that the faint areas would be more visible in the photographs. Scale bars, $10 \mu \mathrm{m}$. 

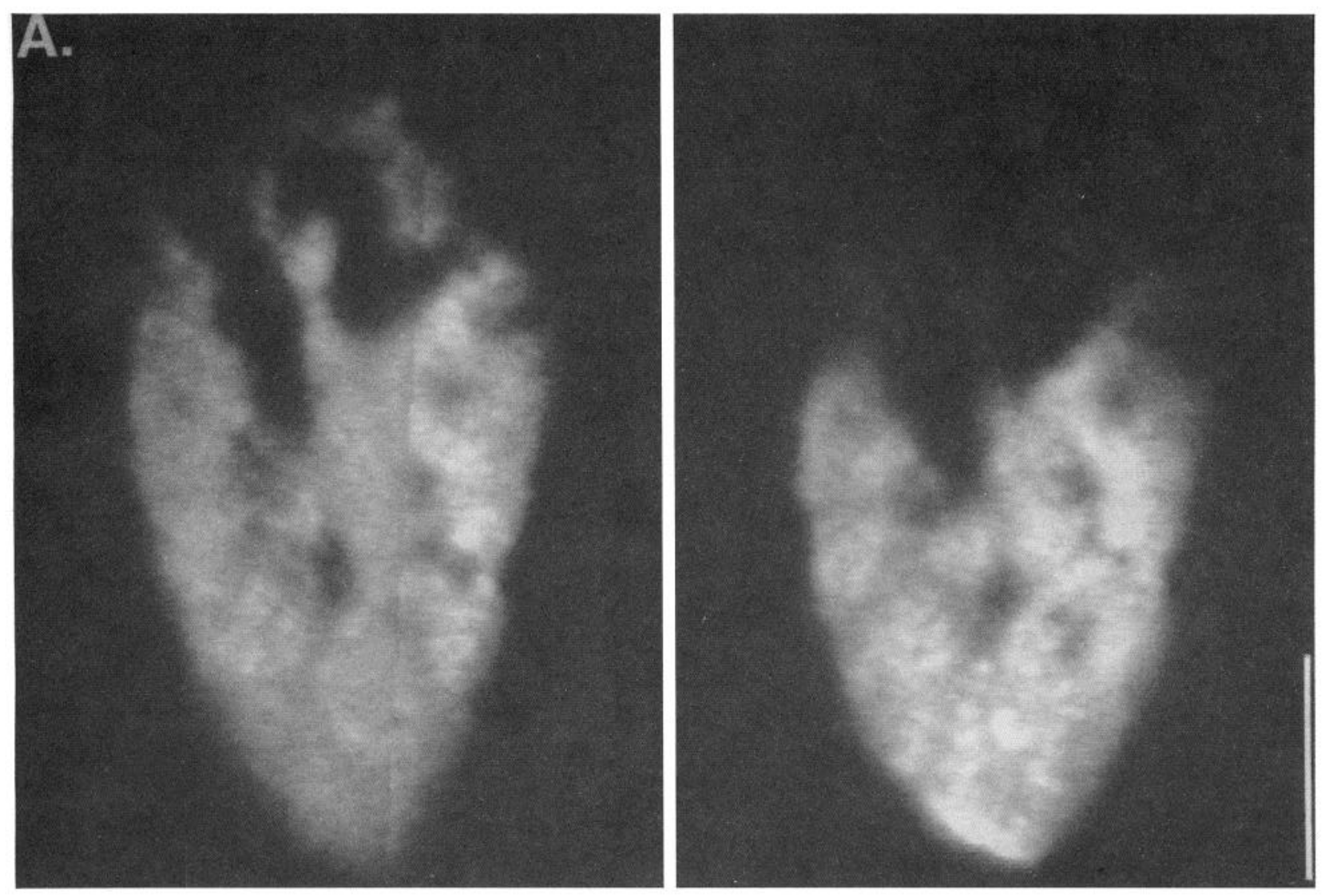

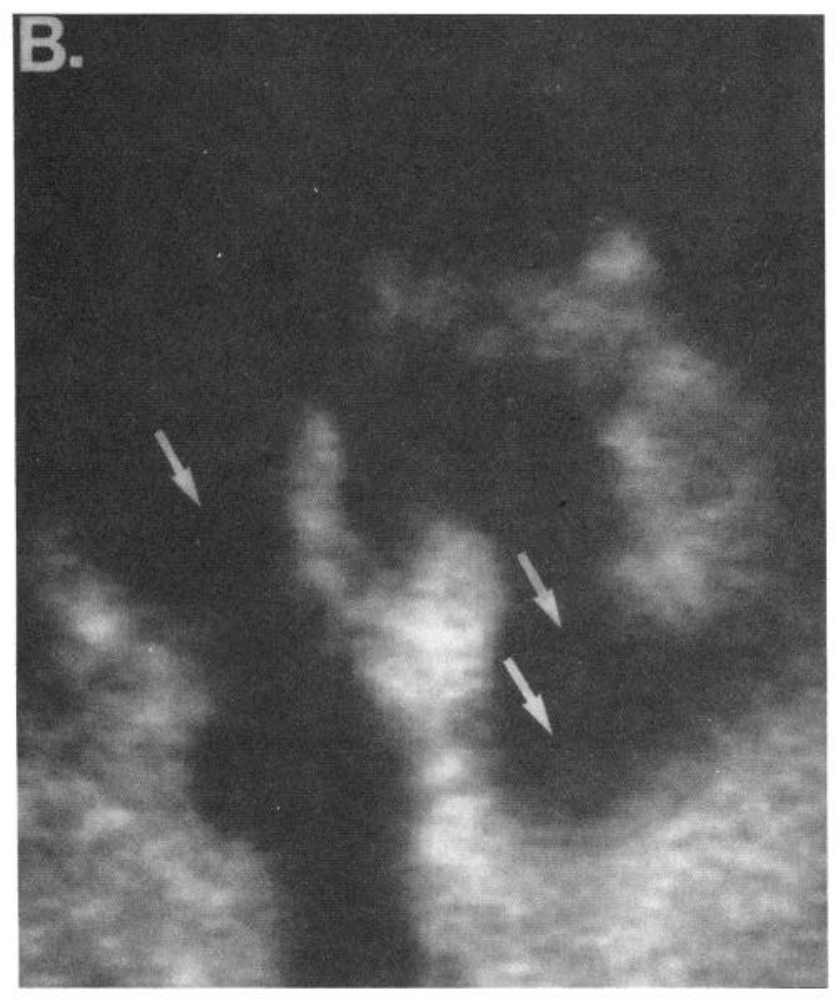

Original $R \propto B T X$

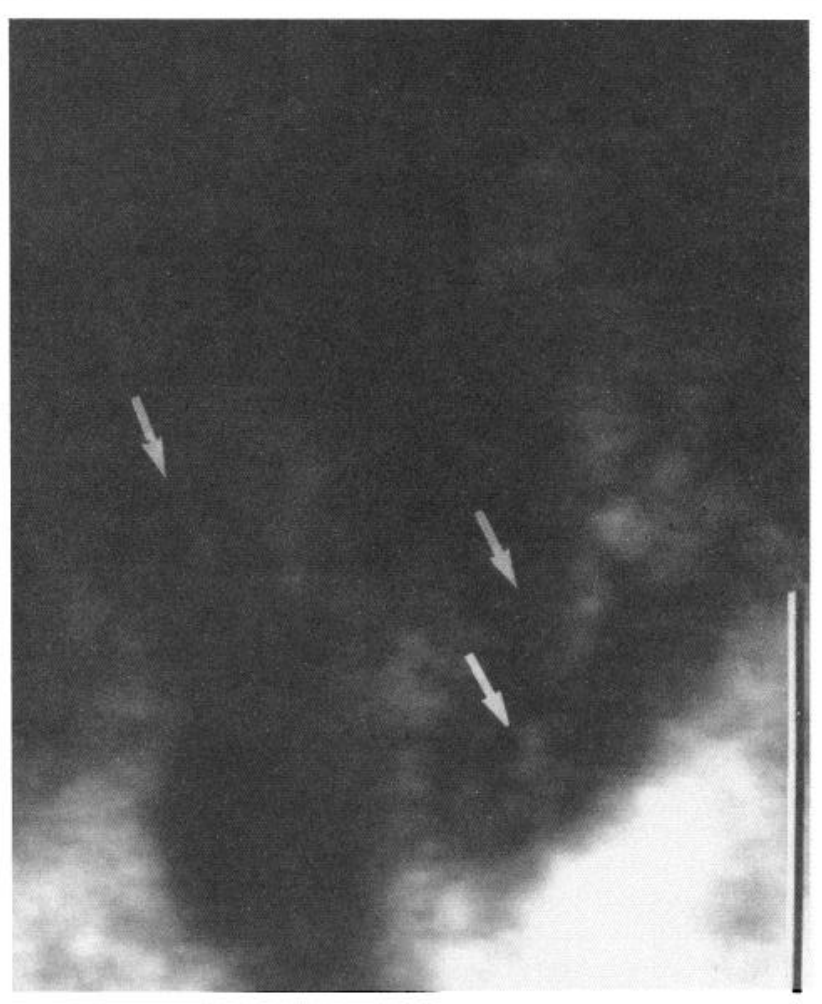

Remaining R $\propto B T X$ 17 hours later 

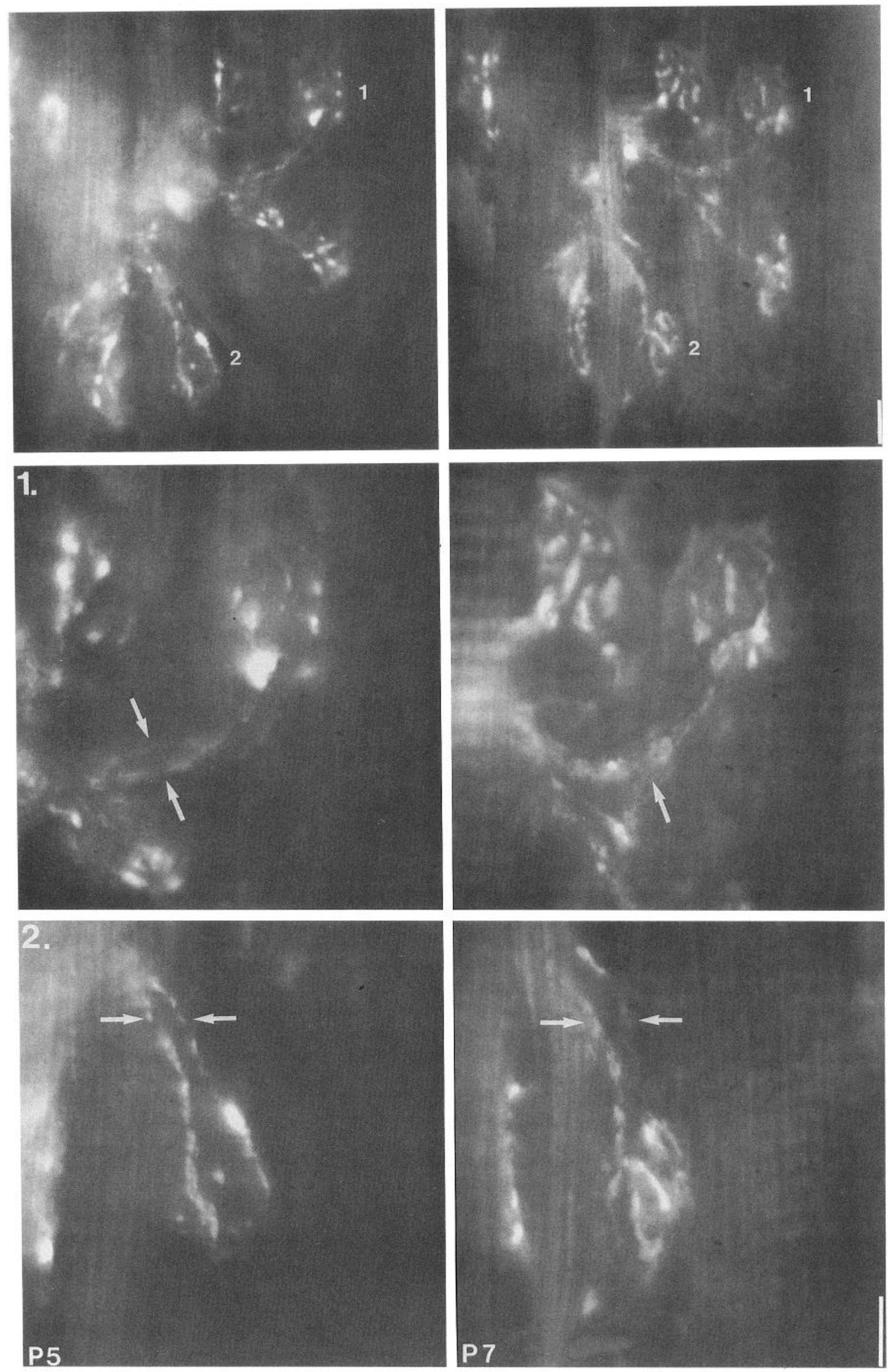

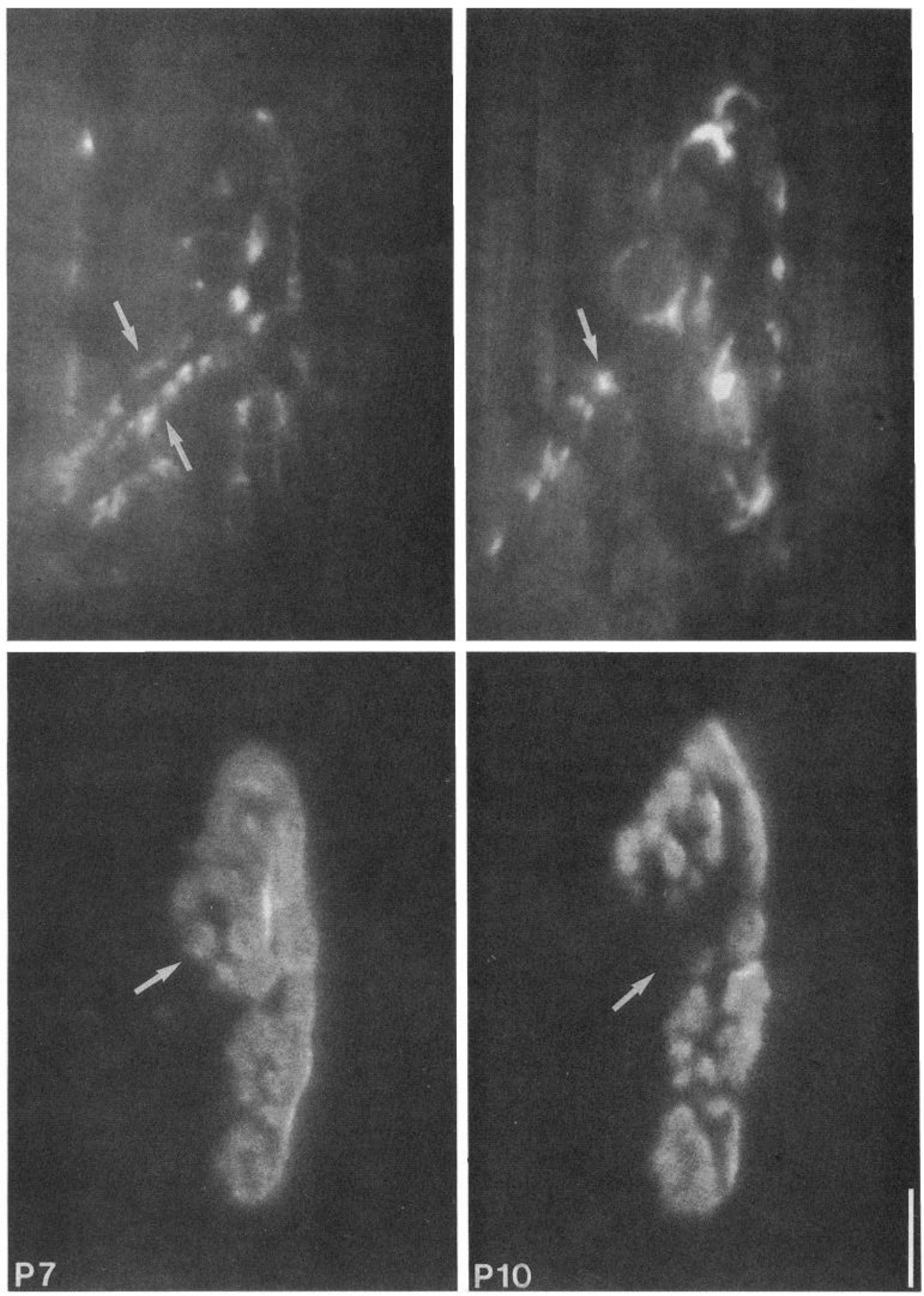

Figure 9. Individual junctions undergoing the transition from multiple to single innervation lose postsynaptic AChR-rich areas. Top, Motor nerve terminals and axons at a junction from a P7 mouse stained with 4-Di-2-ASP. This junction is multiply innervated by two axons (left panel, arrows). The right panel shows the same junction restained and rephotographed $3 \mathrm{~d}$ later, when only one axon remains to singly innervate the junction (right panel, arrow). Bottom, Postsynaptic AChR-rich areas in this junction stained with $\mathrm{R} \alpha \mathrm{BTX}$. AChR-rich areas present on P7 (left panel, arrow) are no longer present when the junction was revisualized on P10 (right panel, arrow). Scale bar, $10 \mu \mathrm{m}$.

that multiply and singly innervated neuromuscular junctions differed dramatically in the incidence of loss of postsynaptic AChR-rich areas.

In every one of the 257 junctions that 4-Di-2-ASP staining showed were innervated by two axons at the first view, AChR- rich areas were observed to be depleted of receptors when the junctions were revisualized several days later (mean, $3 \pm 0.5 \mathrm{~d}$ later; Fig. 9, bottom panels). In 193 of 257 junctions, one of the two axons initially innervating the junction at the first view was no longer present at the second view (Fig. 9, top panels).

Figure 8. Visualization of the transition from multiple to single innervation in the sternomastoid muscle of living mice. Top, Motor axons and nerve terminals from a P5 mouse (left panel) vitally labeled with 4-Di-2-ASP. Shown is a portion of the end-plate band containing five superficial neuromuscular junctions; the bright spots of staining are probably intracellular mitochondria within motor axons and nerve terminals. Muscle fibers are more weakly stained, and some myofibrillar striations can be seen. The right panel shows the same area restained and rephotographed $2 \mathrm{~d}$ later. The junctions labeled 1 and 2 are shown at higher magnification below. Middle, Shown is a neuromuscular junction from a P5 mouse that is innervated by two motor axons that are entwined (left panel, arrows). Two days later, focusing through this region revealed that only one of these axons remains (right panel, arrow). Bottom, Shown is a neuromuscular junction innervated by two parallel motor axons (left panel, arrows). Two days later, one axon has withdrawn from the junction but is still present (right panel, right arrow) adjacent to the preterminal portion of the axon that remains (right panel, left arrow). Scale bars, $10 \mu \mathrm{m}$. 

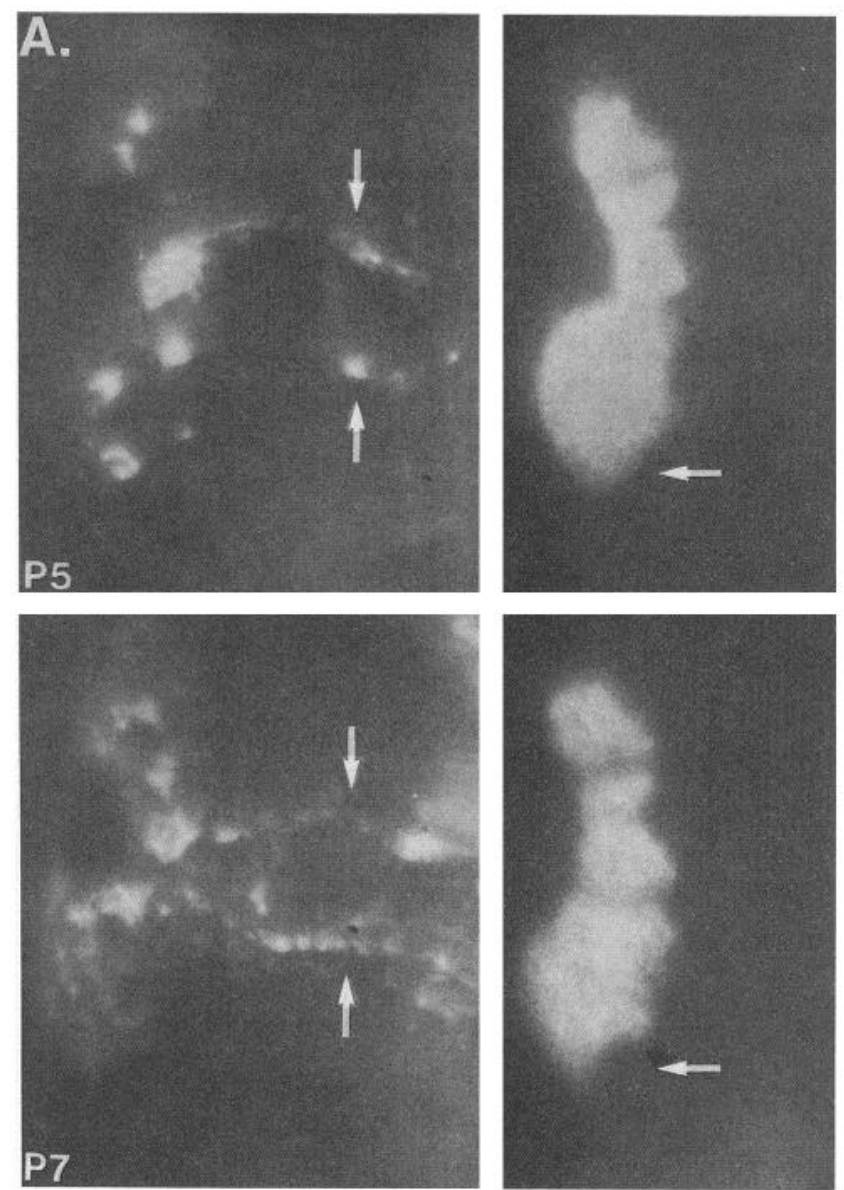
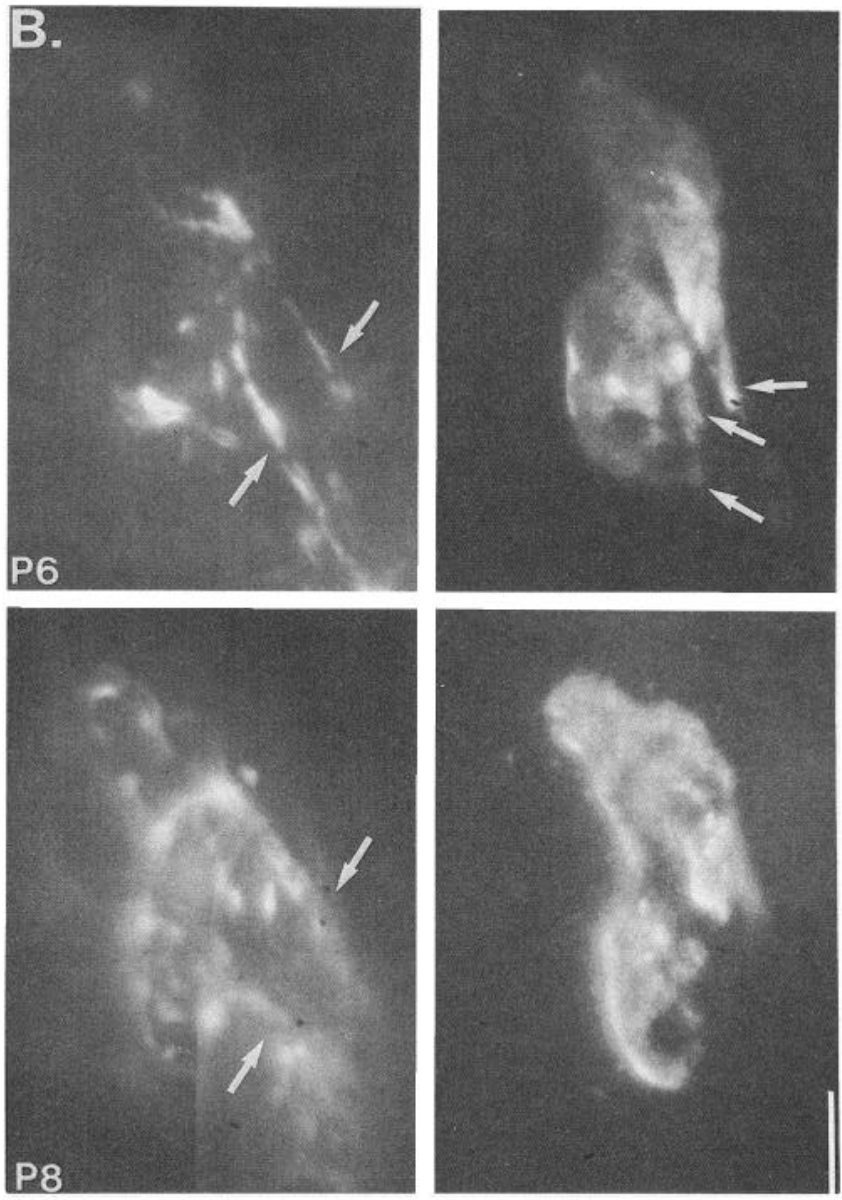

Figure 10. Postsynaptic AChR-rich areas are lost prior to axon withdrawal from multiply innervated junctions. A, Motor nerve terminals and axons in a junction from a P5 mouse stained with 4-Di-2-ASP. The junction is innervated by two motor axons (top left panel, arrows). The top. right panel shows the postsynaptic AChR-rich areas in this junction stained with R $\alpha$ BTX. Bottom panels show the same junction restained and rephotographed on P7. Although the junction remains multiply innervated (bottom left panel, arrows), AChRs have been depleted from a small area of the junction in the interval between views (right panels, arrow). B, Motor nerve terminals and axons in a multiply innervated junction from a P6 mouse stained with 4-Di-2-ASP (top left panel). The top right panel shows the postsynaptic AChR-rich areas in this junction stained with $\mathrm{R} \alpha \mathrm{BTX}$. Bottom panels show the same junction restained and rephotographed on P8. AChRs have been depleted from the bottom portion of the junction in the interval between views (compare top right panel, arrows, with corresponding area in bottom panel). Scale bar, $10 \mu \mathrm{m}$.

In 64 of 257 junctions, both axons remained at the second view. Nonetheless, each of these junctions also contained AChR-rich areas that were depleted of receptors between views (Fig. 10). Thus, loss of AChR-rich areas can occur prior to the withdrawal of a motor axon from a junction.

In contrast, 116 of 119 junctions that appeared singly innervated at the first view showed no evidence of depletion of AChRs from postsynaptic areas when they were viewed a second time several days later (mean, $4 \pm 0.4 \mathrm{~d}$ later; Fig. 11). These results show that depletion of AChRs occurs very frequently in junctions undergoing the transition from multiple to single innervation but relatively rarely in junctions in which axon withdrawal has already taken place. Thus, the large group of junctions described in sections 2 and 3 which did not undergo loss of AChR-rich areas were most likely already singly innervated prior to the time the first view was made.

\section{Motor nerve terminal staining disappears over areas depleted of $\mathrm{AChRs}$}

The observation that depletion of AChRs from postsynaptic areas is occurring in junctions losing multiple innervation raised the obvious question of what is the spatial relationship between pre- and postsynaptic loss. We first determined that when one axon withdrew from a junction, a change in nerve terminal staining is apparent that is consistent with the elimination of sites of nerve terminal contact. Junctions were stained with 4-Di-2-ASP, and those in which the two axons and their nerve terminals appeared to be at least partially segregated within the junction were selected for further study $(N=33$ junctions, 12 mice; mean age at first view, $6 \pm 0.3 \mathrm{~d}$ ). These junctions were viewed a second time a mean of $2 \pm 0.9 \mathrm{~d}$ later.

In 26 of 33 of these junctions, as one axon withdrew, motor nerve terminal staining adjacent to that axon also disappeared. In the examples illustrated in Figure 12, several small 4-Di-2ASP-stained spots near one axon that were present at the first view (left panels, rightmost arrows) were no longer present when the junctions were restained and reviewed $2 \mathrm{~d}$ later (right panels). That areas in fact lost 4-Di-2-ASP staining in each of these junctions at the second view suggests that the remaining axon did not re-occupy those areas (Fig. 12, right panels).

To compare pre- and postsynaptic sites within a junction before and after one axon withdrew, junctions were stained with both 4-Di-2-ASP and $\operatorname{R} \alpha \mathrm{BTX}(N=124$ junctions, 27 mice; mean age at first view, $6 \pm 0.5 \mathrm{~d}$; second view, mean of $3 \pm$ 

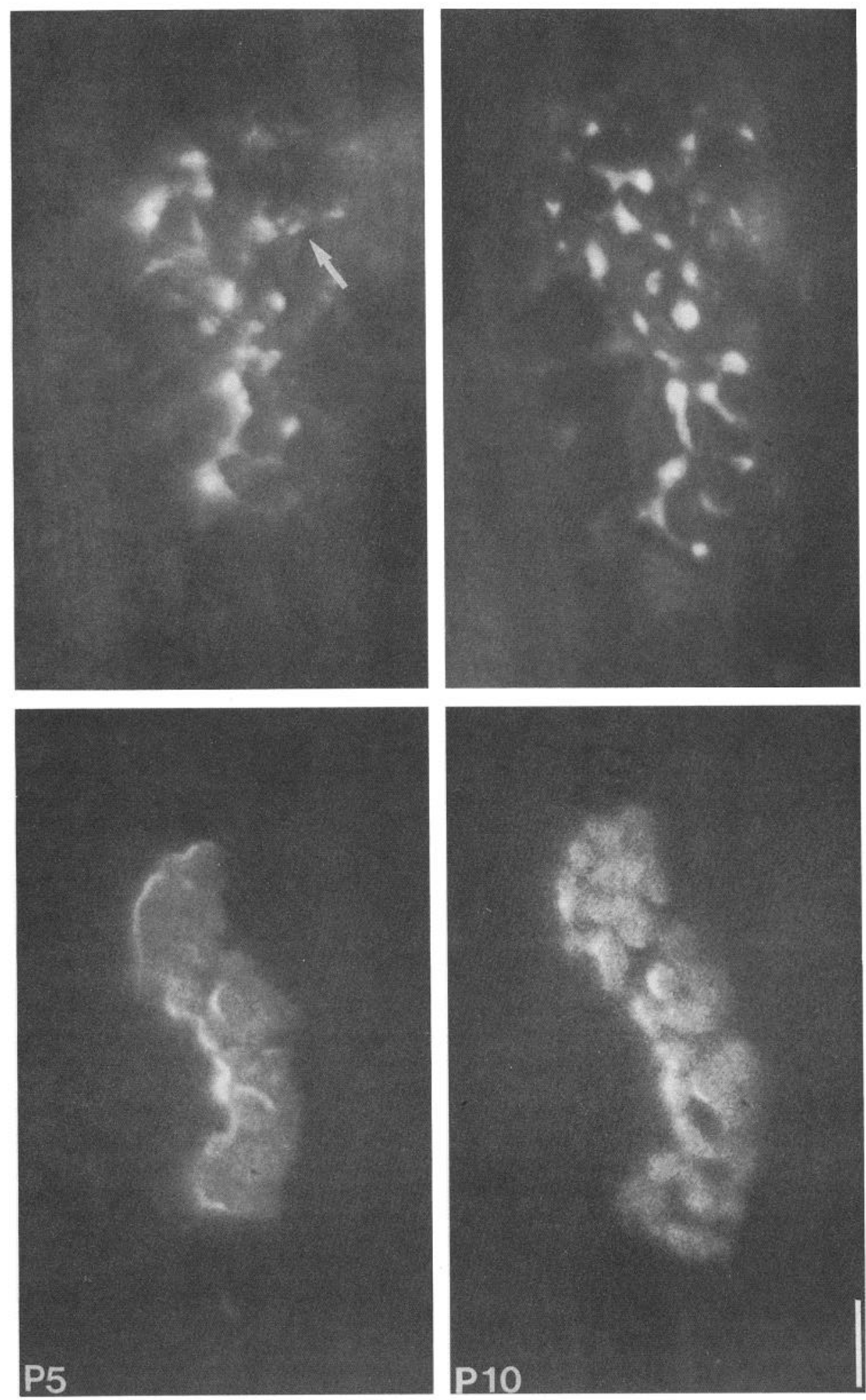

Figure 11. AChR-rich areas in singly innervated junctions spread apart as junctions grow but are otherwise unchanged. Top, Motor nerve terminals in a junction from a P5 mouse (left pan$e l$ ) stained with 4-Di-2-ASP. This junction is singly innervated by one motor axon (arrow) whose full length is not visible in the single focal plane illustrated here. The spots of staining above and below this axon are probably mitochondria in Schwann cells that begin to ensheath axons at these ages. The right panel shows the same junction restained and rephotographed on P10. Bottom, Postsynaptic distribution of AChR-rich areas in this junction stained with $\mathrm{R} \alpha \mathrm{BTX}$. The junction enlarged by spreading apart of existing postsynaptic $\mathrm{AChR}$-rich areas in the interval between views. As this occurred, gaps became apparent in formerly compact receptor-rich areas. In contrast to multiply innervated junctions undergoing synapse elimination at the same ages, no AChR-rich areas completely disappeared from singly innervated junctions in the interval between views. Scale bar, $10 \mu \mathrm{m}$.

$0.3 \mathrm{~d}$ later). In every case where postsynaptic areas were depleted of AChRs, motor nerve terminal staining was lost from the same synaptic areas that were depleted of AChRs (Fig. 13, uppermost arrow). Depletion of AChRs was not observed from areas of the junction that maintained presynaptic staining.

On the other hand, areas that lost 4-Di-2-ASP staining did not always also lose postsynaptic AChRs (see Fig. 13, arrow- heads). Because 4-Di-2-ASP only stains intracellular organelles such as mitochondria (Lichtman et al., 1989), one possibility is that loss of 4-Di-2-ASP staining may merely represent mitochondrial rearrangements within the nerve terminal rather than actual loss of a nerve terminal per se. However, in neuromuscular junctions stained with RH795 (which intercalates into the motor nerve terminal membrane; Grinvald et al., 1986), the 
Figure 12. Areas of motor nerve terminal staining disappear during the transition from multiple to single innervation. $A$, Motor nerve terminals and axons stained with 4-Di-2-ASP in a junction from a P5 mouse multiply innervated by two motor axons (left panel, upper arrows). The right panel shows the same junction restained and rephotographed on P7, when one axon remains (arrow). In addition to the withdrawal of one motor axon from the upper right-hand area of the junction, motor nerve terminal staining has also disappeared from a nearby area (compare left panel, rightmost arrow, with corresponding area in right panel). On P7, mitochondria in an ensheathing Schwann cell are evident surrounding the remaining motor axon. $B$, Motor nerve terminals and axons stained with 4-Di-2-ASP in a junction from a P6 mouse multiply innervated by two motor axons (left panel, leftmost arrows). The right panel shows the same junction restained and rephotographed on $\mathrm{P} 8$, when one axon remains (arrow); this axon is ensheathed by a Schwann cell whose mitochondria are evident surrounding the preterminal portion of the axon. Motor nerve terminal staining has disappeared from the top portion of the junction (compare left panel, rightmost arrow, with corresponding area in right panel). Scale bar, $10 \mu \mathrm{m}$.
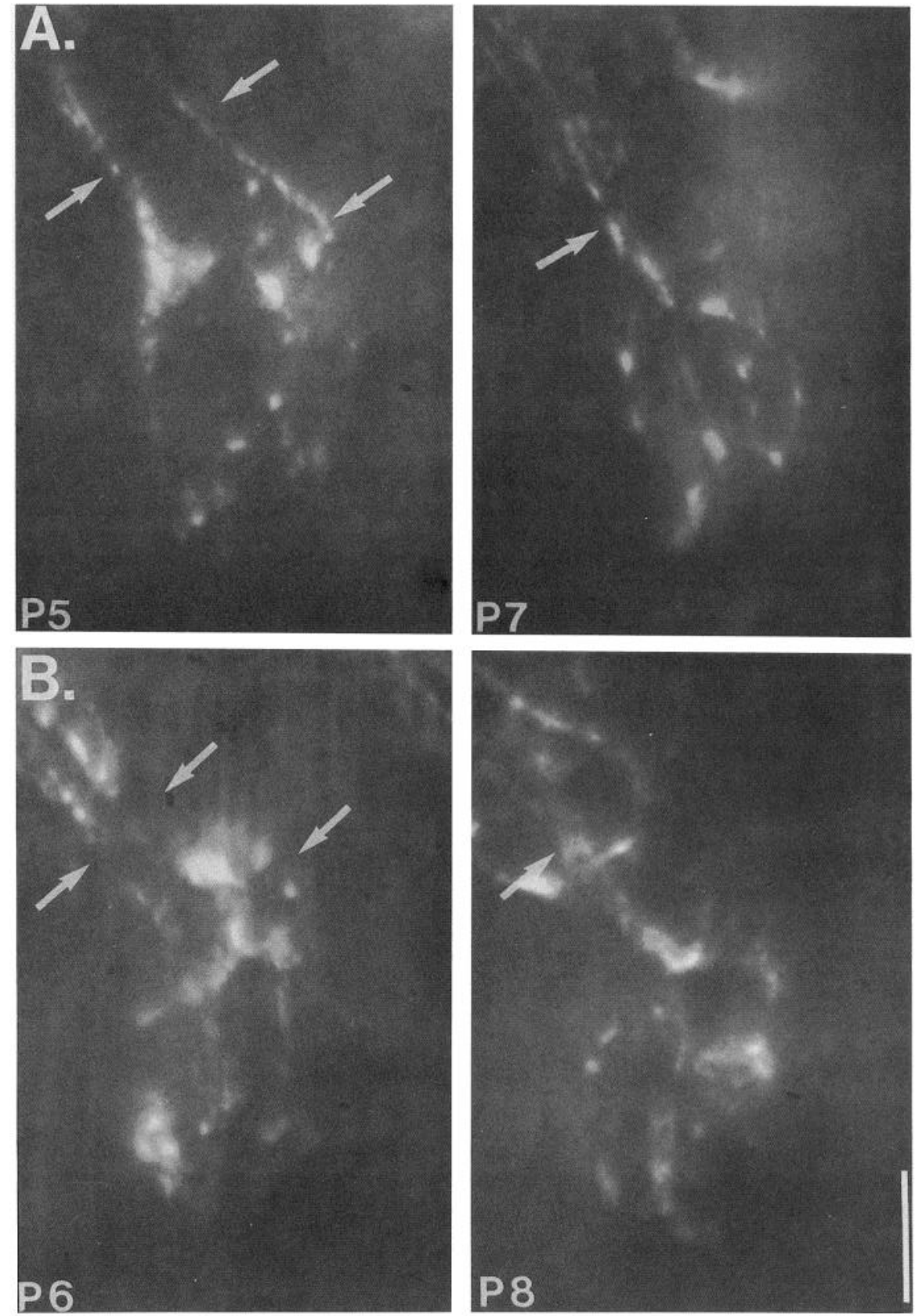

nerve terminal membrane (contiguous with the motor axon) aligned exactly with brightly stained postsynaptic AChR-rich areas at all of the ages we examined ( $N=79$ junctions, 30 P4P14 mice; see Figs. 15, 16). Thus, as AChR-rich areas are lost during development, the overlying nerve terminal must also be eliminated.

\section{AChR depletion begins before the overlying motor nerve terminal is eliminated}

One question raised by these observations is whether the loss of postsynaptic AChR-rich areas occurs after and perhaps as a consequence of motor nerve terminal elimination, or alternatively, whether elimination of motor nerve terminals is a consequence of postsynaptic changes, including depletion of AChRs. To address this question, it was necessary to determine more precisely when motor nerve terminals disappeared relative to when AChRs in the underlying membrane began to be depleted. By looking at faintly stained AChR areas that were in the process of being eliminated, we could ask if they consistently were no longer overlain by nerve terminal staining, which would indicate that loss of AChR staining occurs after the nerve has already vacated those areas. Examination of multiply innervated junctions stained with 4-Di-2-ASP and R $\alpha$ BTX that contained faint AChR areas ( $N=89$ junctions, 27 P6-P8 mice), however, showed that AChR loss often occurred prior to loss of motor nerve terminal staining, because faint receptor areas were still overlain by 4 -Di-2-ASP staining ( 78 of 89 junctions; Fig. 14). This indicates that 4-Di-2-ASP staining is not eliminated until after AChRs begin to be depleted postsynaptically. By staining with 4-Di-2-ASP as well as RH795 to label the membrane of the nerve terminals, we could determine whether or not the 4-Di2-ASP staining remaining over areas of low AChR density was 

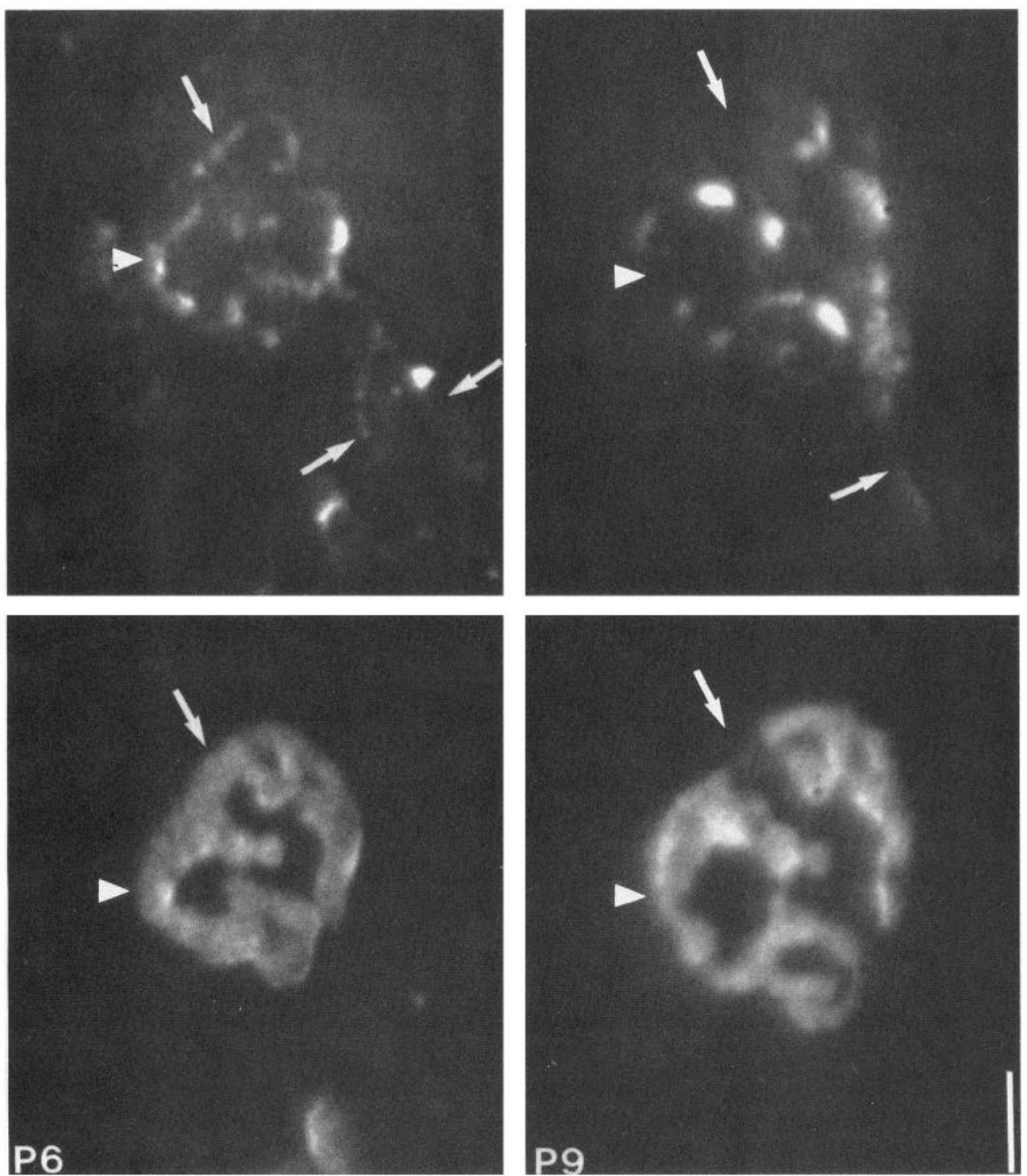

Figure 13. Motor nerve terminals are eliminated from junctional areas that lose postsynaptic AChRs. Top, The left panel shows motor nerve terminals and axons in a junction from a P6 mouse stained with 4-Di-2-ASP. This junction is multiply innervated by two axons (lower arrows). The right panel shows the same junction restained and rephotographed on $\mathrm{P} 9$, when one axon remains (lower arrow). In addition to the withdrawal of one axon, the pattern of motor nerve terminal staining has changed in the interval between views. One of these changes is the loss of 4-Di2-ASP staining from the upper region of the junction (upper arrows). A second change is the loss of staining from the left-hand region of the junction (arrowhead). Bottom, Postsynaptic AChR-rich areas in this junction stained with $\mathrm{R} \alpha \mathrm{BTX}$. The postsynaptic area beneath a site of loss of 4-Di-2-ASP staining has been depleted of AChRs (compare right panel, arrow, with left panel, arrow). Because changes in 4-Di-2-ASP staining did not always correspond with changes in AChR staining (compare preand postsynaptic sites marked by arrowheads in top and bottom panels), we also stained junctions with a vital dye that labeled motor nerve terminal membranes (see Fig. 15). Scale bar, 10 $\mu \mathrm{m}$.

part of the nerve terminal. We compared the pattern of staining of multiply innervated junctions with 4-Di-2-ASP and RH795 at various stages of synapse elimination $(N=154$ junctions, 65 P6-P8 mice).

In each of the multiply innervated junctions in which postsynaptic AChR areas were stained uniformly brightly with $\operatorname{R} \alpha \mathrm{BTX}$ ( $N=113$ junctions), the postsynaptic area was entirely overlain by RH795 stained motor nerve terminals and the RH795 staining enveloped 4-Di-2-ASP staining (Fig. 15, top row). However, in 35 of 41 multiply innervated junctions in which postsynaptic areas stained faintly with $\mathrm{R} \alpha \mathrm{BTX}$, the faint AChR areas still had RH795 and 4-Di-2-ASP stained motor nerve terminals over them (Fig. 15, middle row). This result not only confirms that depletion of $\mathrm{AChRs}$ can occur before a motor axon entirely withdraws from a junction (see section 4 and Fig. 10), but also shows that depletion of receptors begins before an overlying motor nerve terminal is eliminated from that site. In 6 of 41 junctions, faintly stained AChR areas were unoccupied or only partially overlain by nerve terminal staining, suggesting that after AChRs began to disappear from a region, nerve terminal staining is short-lived (Fig. 15, bottom row).

\section{Loss of synaptic sites is rare after single innervation is established}

Studies in which neuromuscular junctions in other mammalian muscles (Wigston, 1989, 1990; Hill et al., 1991) and in other species (Herrera et al., 1990; Chen et al., 1991) were followed over time have suggested that loss of synaptic sites may occur throughout life. Because junctions in adult skeletal muscles are assumed to be uniformly singly innervated (at least in mammals), this raises the possibility that there are causes other than the presence of two (or more) competing inputs for pre- or postsynaptic loss. To study further the relative incidence of loss of synaptic regions from junctions in the sternomastoid muscle after the period of synapse elimination and to understand better the cause, we followed junctions stained with RH795, 4-Di-2ASP, and R $\alpha$ BTX during subsequent maturation ( $N=39$ junctions, 121 -month-old mice; junctions viewed at roughly 1 month intervals; 26 junctions viewed three times, 12 viewed four times, 1 viewed five times). In most junctions (35 of 39), no postsynaptic AChR-rich areas or presynaptic motor nerve terminals were observed to be lost when junctions were followed for up 
Figure 14. Postsynaptic areas begin to be depleted of AChRs before overlying motor nerve terminals are eliminated. Shown are two examples $(A, B)$ of neuromuscular junctions in sternomastoid muscles from P7 mice that were stained with 4-Di-2-ASP (left panels) and $\mathrm{R} \alpha \mathrm{BTX}$ (middle panels). These junctions are multiply innervated by two motor axons (left panels, arrowheads). Within each junction there are areas of $\mathrm{R} \alpha \mathrm{BTX}$ staining that are fainter than the remainder of the junction (middle panels, arrows) and that are in the process of being depleted of AChRs. A "bleedthrough" image (see Materials and Methods) of 4-Di-2-ASP staining plus $\mathrm{R} \alpha \mathrm{BTX}$ staining taken with rhodamine filters shows that these faintly stained areas are overlain by motor nerve terminal staining (compare left, middle, and right panels, arrows). Scale bar, $10 \mu \mathrm{m}$.
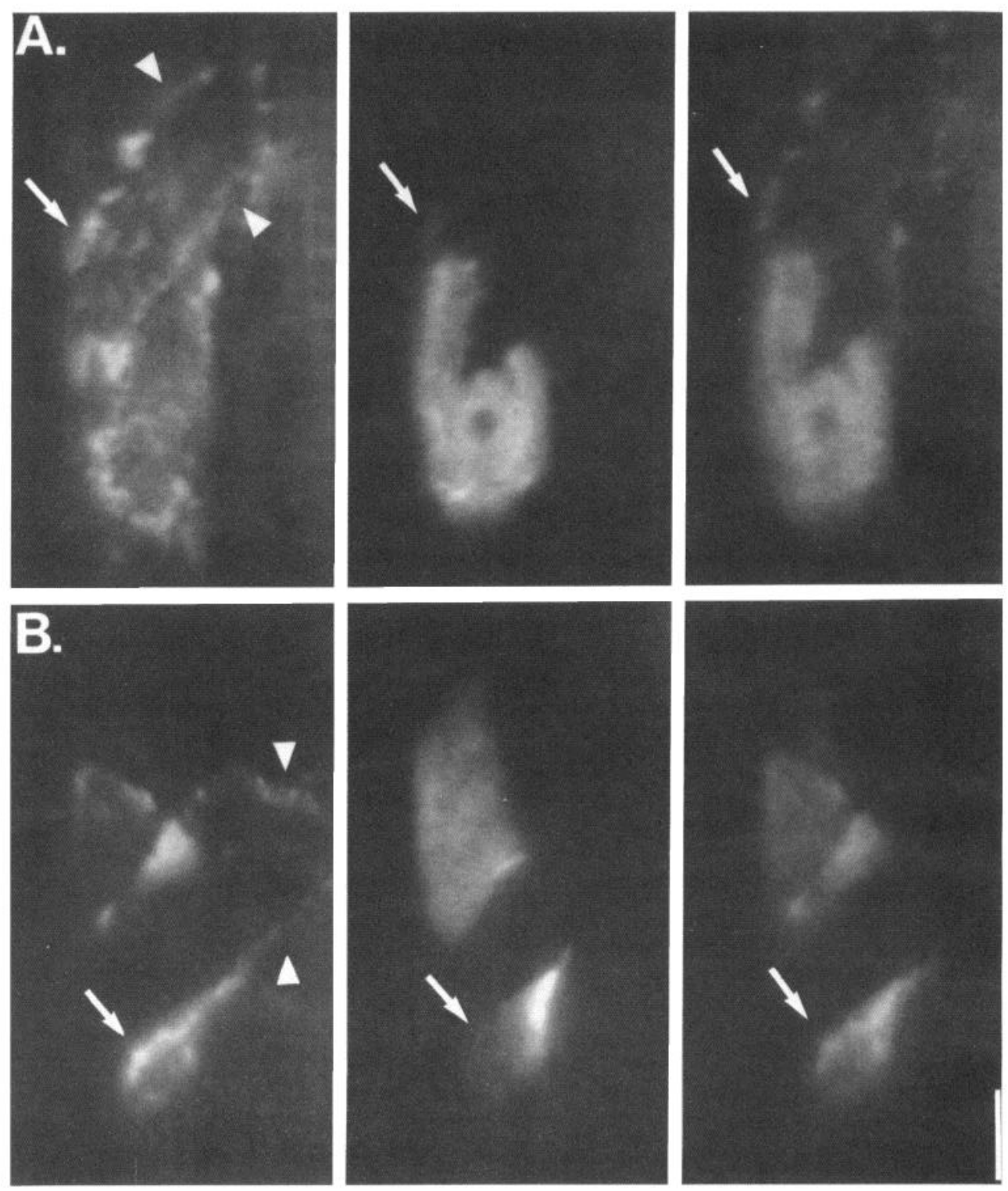

to 7 months (see also Balice-Gordon and Lichtman, 1990b, their Figs. 7, 8, 11, 12; Balice-Gordon and Lichtman, 1991, their Figs. 1, 2). In 4 of 39 junctions, however, synaptic areas disappeared over the time the junction was observed. These areas were typically small, less than $40 \mu \mathrm{m}^{2}$ in area (compared to an average junctional area of $1296 \pm 319 \mu \mathrm{m}^{2}$ at 1 month of age). One of these junctions is illustrated in Figure 16. Over a 5 month interval, three small AChR-rich areas were observed to be lost from the junction. Between 1 and 4 months of age, a small gap appeared in the upper left-hand region of this junction (top right panel, arrow) and similarly sized gaps appeared in two other junctions. It is possible, however, that the appearance of such gaps is an example of spreading apart of adjacent receptor areas due to growth rather than true loss of a synaptic region (see

Figure 15. Alignment of pre- and postsynaptic regions before, during, and after synapse elimination. Each row illustrates a neuromuscular junction in which motor nerve terminals and axons were stained with 4-Di-2-ASP (left panels), RH795 (middle panels); postsynaptic AChR-rich areas were stained with $\mathrm{R} \alpha \mathrm{BTX}$ (right panels). Top, Shown is a multiply innervated neuromuscular junction from a P5 mouse in which all of the AChRrich areas are occupied by motor nerve terminal staining. Middle, Shown is a multiply innervated neuromuscular junction from a P7 mouse that contains a postsynaptic area that is partially depleted of AChRs (right panel, arrow). This area stains more faintly than the other AChR-rich areas in the junction. Motor nerve terminals, stained with 4-Di-2-ASP (left panel, arrow) or RH795 (middle panel, arrow) not only are present over the brightly stained AChR-rich areas but also are present over the faintly stained area. Observations of junctions such as this one (see also Fig. 14) suggested that AChRs begin to be depleted from a postsynaptic area before the overlying motor nerve terminals are eliminated and the motor axon withdraws from the junction. Bottom, Shown is a junction from a P8 mouse that is innervated by a well-stained axon (left and middle panels). There may also be a faintly stained remnant of a second axon entering the junction above the well-stained axon that could be traced back to an intramuscular nerve branch. Brightly stained areas of AChRs are occupied by motor nerve terminal staining, and a faint AChR area at the top of the junction appears to be unoccupied by nerve terminal staining. This suggests that after AChRs begin to disappear from a region, nerve terminal staining over that region is short-lived. Note the more highly branched AChR distribution in this junction from a P8 mouse, which is typical of singly innervated junctions (compare right panels in each row). Scale bar, $10 \mu \mathrm{n}$. 

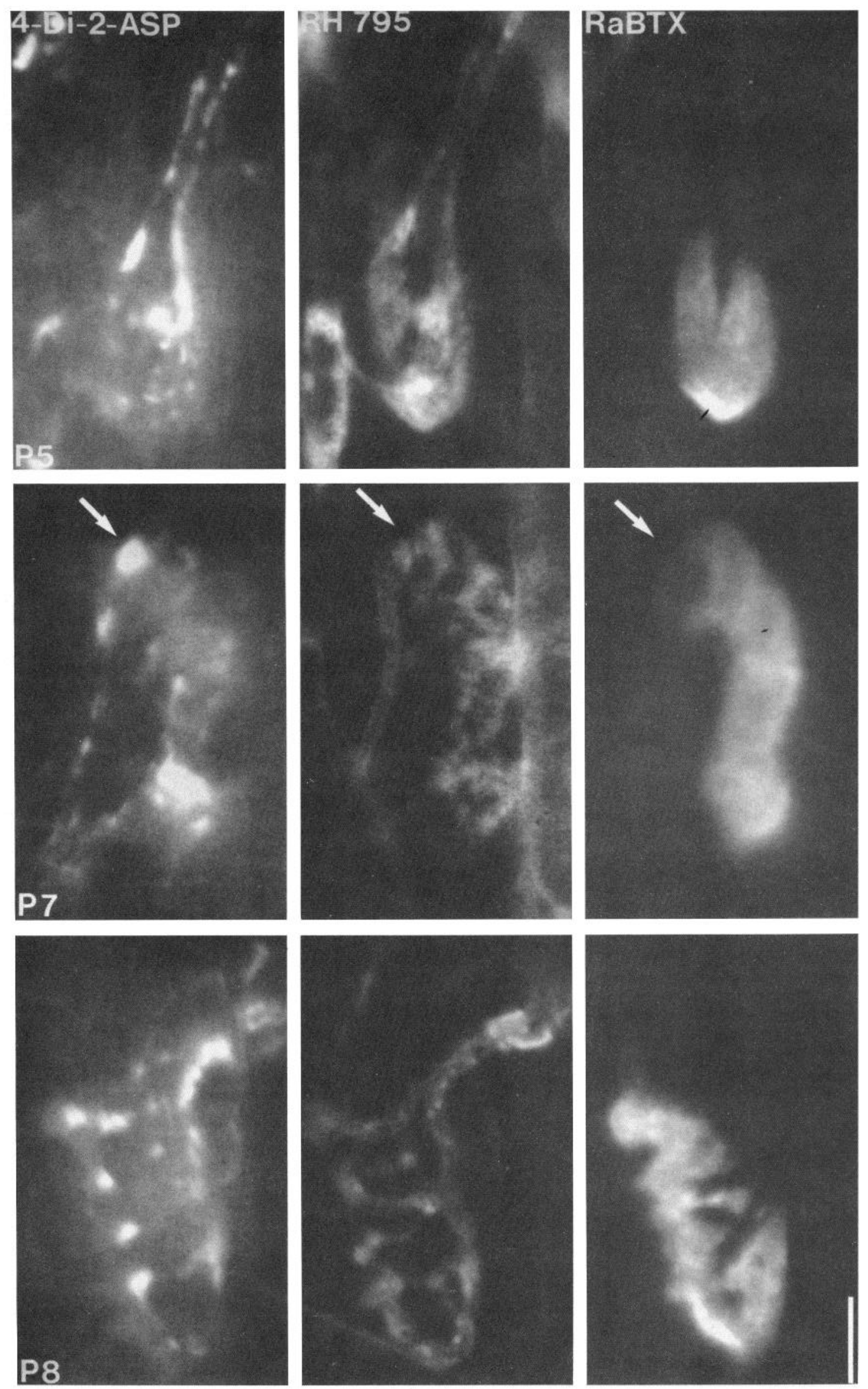

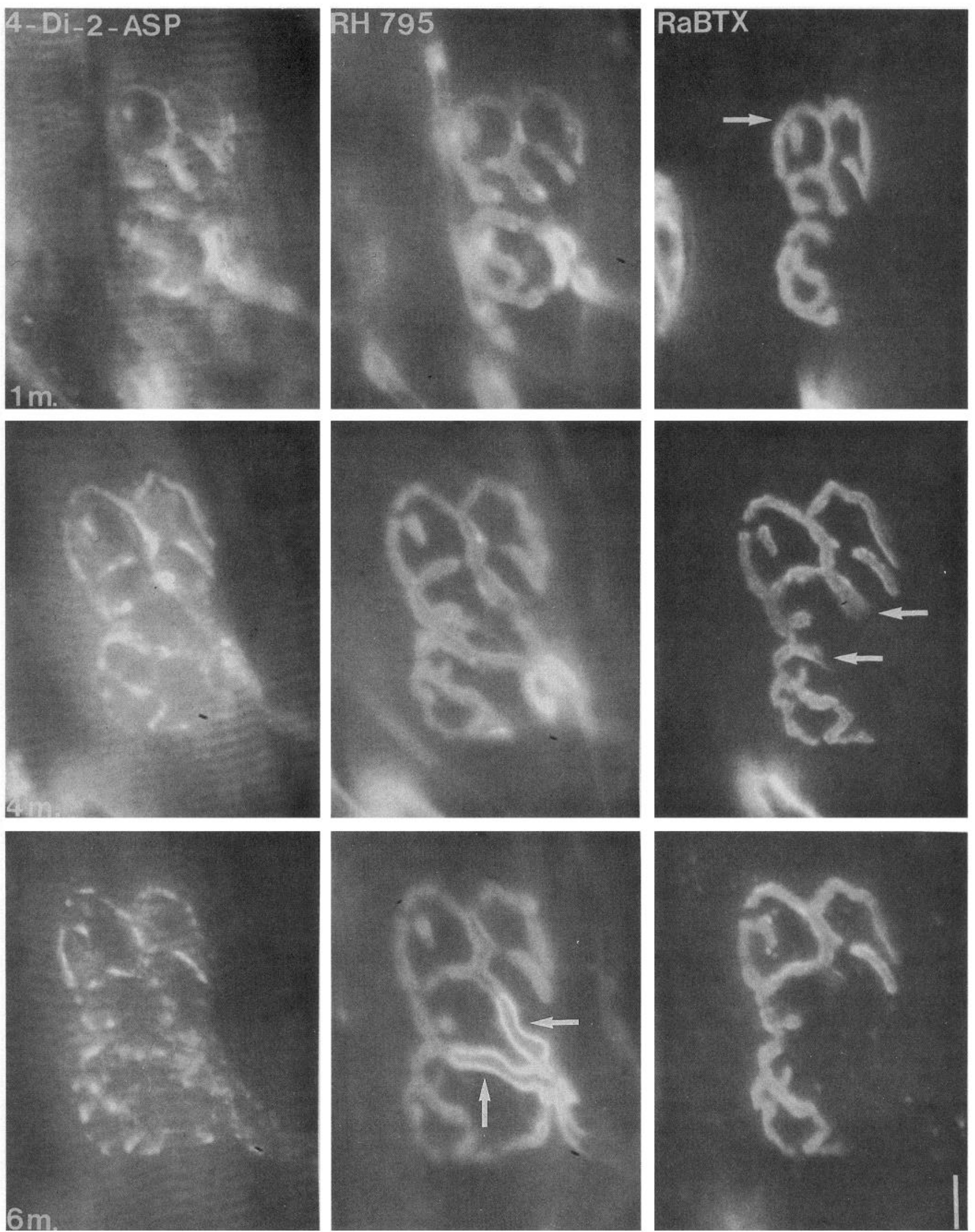

Figure 16. Loss of synaptic areas is only occasionally observed in sternomastoid neuromuscular junctions after single innervation is established in the muscle. Shown are 4-Di-2-ASP staining (left panels), RH795 staining (middle panels), and R $\alpha$ BTX labeling (right panels) of a junction in a sternomastoid muscle from a 1 -month-old mouse $(1 \mathrm{~m}$.) that was restained and rephotographed at 4 months $(4 \mathrm{~m}$.) and $6 \mathrm{months}(6 \mathrm{~m}$.) of age. RH795 staining shows the outline of terminal and axon membrane, which completely encloses 4-Di-2-ASP staining. Presynaptic motor nerve 
section 2). Between 4 and 6 months of age, two other AChRrich areas in this junction have been depleted of receptors (middle left panel, arrows). By viewing the RH795-stained nerve terminals at the same time points, it is clear that in this case, as well as a similar loss of a receptor-rich area in one other junction, the loss is related to the myelination of a former nerve terminal branch (bottom middle panel, arrows).

These observations show that in the mouse sternomastoid muscle, ongoing loss of pre- or postsynaptic areas in singly innervated junctions is relatively rare after the period of synapse elimination is completed. Moreover, the synaptic sites that remain after synapse elimination is complete are stable for many months. Thus, the synaptic sites that are lost give rise to changes in the junctional branching pattern that are maintained over long intervals.

\section{Discussion}

We have attempted to analyze the changes that occur in mouse neuromuscular junctions during early postnatal life when muscle fibers undergo the transition from multiple to single innervation. The principal findings are that (1) postsynaptic AChRs are commonly depleted from areas of neuromuscular junctions during early postnatal life but not later. This depletion is much more frequently seen in junctions that are innervated by multiple motor axons than in junctions that are singly innervated. (2) More than one postsynaptic area is depleted of AChRs within each junction. These losses occur in succession rather than simultaneously. (3) Nerve terminals are eliminated presynaptically from the same areas that are depleted of AChRs postsynaptically. (4) Presynaptic nerve terminal loss does not occur until after postsynaptic AChRs begin to be depleted, and (5) this pre- and postsynaptic loss permanently changes the branching pattern of a junction. From these results, we conclude that the withdrawal of a motor axon from a neuromuscular junction occurs as a consequence of the sequential elimination of all of its synapses within that junction. It appears that one axon begins to lose the battle for exclusive innervation of a junction as the postsynaptic areas it occupies are depleted of AChRs. Thus, the postsynaptic target cell may play an active role in deciding which synapses are to be maintained and which are to be eliminated.

One weakness in this work is that the quality of the vital presynaptic staining was usually insufficient to identify the axonal source of the presynaptic terminals that were eliminated. We cannot say with certainty that the nerve terminal staining that was seen to disappear always originated from the axon that ultimately was eliminated. In a few optimally stained junctions (see Fig. 14; Fig. 15, middle row), motor nerve terminals over a faintly stained AChR area could be seen to originate from the vicinity of one axon, arguing that the area being lost was associated with that axon. In other work (Balice-Gordon and Lichtman, 1989; Chua et al., 1990; R. J. Balice-Grordon, C. K. Chua, and J. W. Lichtman, unpublished observations), we have approached this problem more directly by using nonvital staining techniques, which show the full extent of each of the terminal arbors at multiply innervated muscle fibcrs by staining cach of them with a different-colored fluorescent dye. That work indicates that prior to axon withdrawal there is a gradual loss of synaptic territory occupied by one axon. The present results are consistent with this idea, and add to this conclusion by showing that there are a number of sites of postsynaptic loss that correspond to sites of motor nerve terminal elimination. However, we still do not know whether some of the symaptic sites that are eliminated belong to the axon that will remain. It seems likely that the losing axon must undergo the majority (perhaps all) of the synaptic loss, which ceases only when the losing axon finally withdraws. The low level of synapse loss observed at singly innervated junctions seems to be due to small changes in the maturing nerve terminal such as myelination of former terminal branches.

If withdrawal of a motor axon from a neuromuscular junction is the consequence of the loss of its synapses, these results suggest that there is a temporal disjunction between these two phenomena. This may mean that in certain situations in which the number of axons innervating a target cell does not change, changes in synaptic input to that cell could occur by the elimination of some of the synapses of belonging to an axon. Because individual synaptic sites were lost over short times (less than 2 d; see Figs. $4,5,7$ ), this form of synaptic plasticity could be involved in rapid changes that occur in the nervous system as a result of synaptic competition.

The present results offer some clues about the mechanism of synapse elimination. The fact that postsynaptic sites are lost with such rapidity is inconsistent with the idea that postsynaptic loss is merely the consequence of the loss of the overlying terminal. Vacated postsynaptic sites in developing neuromuscular junctions have been observed to lose their morphological characteristics, but this occurs over longer periods ( $>1$ week; Slater, $1982 \mathrm{~b}$ ). Moreover, the postsynaptic areas that will be lost begin to be depleted of AChRs in advance of the structural withdrawal of the overlying nerve terminal. Thus, in both development and reinnervation (Rich and Lichtman, 1989a), loss of AChR-rich areas occurs prior to the structural elimination of the nerve terminal. The early postsynaptic changes suggest a possible role for the postsynaptic cell both in eliminating and perhaps also maintaining a presynaptic nerve terminal.

Previous work in the sternomastoid and bulbocavernosus muscles has made the case that nerve terminals are adherent to the postsynaptic cell at synaptic sites (Balice-Gordon and Lichtman, 1990b, 1991; Balice-Gordon et al., 1990). This adhesion seems to be the basis for both the intercalary enlargement of nerve terminals in muscles during growth as well as the maintenance of precise alignment of pre- and postsynaptic sites during growth. If such adhesion is also present during the first 2 postnatal weeks, then synapse elimination not only dismantles the postsynaptic apparatus, but also must break the adhesive bond the nerve terminal makes with the postsynaptic membrane (via the basal lamina). We do not know what kind of causal link exists between loss of postsynaptic AChR-rich areas and nerve

terminals were observed to align precisely with postsynaptic AChR-rich areas. Over the 5 months this junction was studied, three small postsynaptic AChR-rich areas disappeared. Between 1 month and 4 months of age, a small gap appears in the upper left-hand region of the junction (top right panel, arrow), probably due to the spreading apart of adjacent postsynaptic areas as the muscle fiber grows (Balice-Gordon and Lichtman, 1990b). Between 4 months and 6 months of age, two small receptor-rich areas are lost (middle right panel, arrows) as the overlying nerve terminal branch became myelinated and thus stained much more intensely (bottom middle panel, arrows). Loss of synaptic regions after single innervation is established is far more infrequent than the loss observed in multiply innervated junctions over the first 2 postnatal weeks. Scale bar, $20 \mu \mathrm{m}$. 
tcrminal elimination, but these phenomena seem to be closely associated. Loss of postsynaptic AChR-rich areas during development, reinnervation (Rich and Lichtman, 1989a), and muscle degeneration (Rich and Lichtman, 1989b) in each case precedes loss of the overlying presynaptic nerve terminal, whereas the converse is not always true: in adult animals presynaptic nerve terminal loss as a consequence of denervation does not cause postsynaptic areas to disappear (Rich and Lichtman, 1989a). Taken together, these results argue that factors derived from the postsynaptic cell are necessary to maintain the overlying nerve terminal actively, whereas presynaptic maintenance of postsynaptic sites may require less active maintenance.

Two questions raised by this work are the following. (1) By what mechanism does the postsynaptic cell signal the nerve terminal that it should withdraw? Is it possible that loss of adhesion is sufficient to cause nerve terminal loss? (2) How do postsynaptic AChR-rich areas come to lose receptors? The present work suggests part of the answer to the second question. We have learned that existing (previously inserted) AChRs are quickly removed from postsynaptic areas undergoing depletion. In views of the same junction obtained over relatively short intervals (hours), AChRs from areas in the process of disappearing occasionally seemed to undergo migration in the membrane between views (see Fig. 7, arrows). More recent efforts using focal labeling with R $\alpha$ BTX (Balice-Gordon and Lichtman, 1990a, and unpublished observations) and fluorescence recovery after photobleaching (Brown et al., 1992; A. P. Brown, P. van Mier, and J. W. Lichtman, unpublished observations) show that even in adult neuromuscular junctions, postsynaptic AChRs are capable of movement within the postsynaptic membrane. If AChRs were more mobile in areas of the postsynaptic membrane in the process of being depleted of receptors than elsewhere within the junction, then receptors might migrate away from one synaptic site and perhaps be trapped by the remaining synaptic sites. Such a mechanism has been invoked to explain AChR clustering (Stollberg and Fraser, 1990), and it may turn out that during synapse elimination axons are competing for AChRs that are "declustering."

Because withdrawal of axons is widespread during neural development, it is possible that the prc- and postsynaptic loss described here is the basis of analogous phenomena in other parts of the nervous system. If this is the case, then loss of synaptic connections generally may require both an early postsynaptic change, including a rapid removal of postsynaptic molecules, and a secondary presynaptic change, which might include the breaking of an adhesive bond between the presynaptic nerve terminal and the postsynaptic apparatus.

\section{References}

Balice-Gordon RJ, Lichtman JW (1989) Competing motor nerve terminals and the acetylcholine receptors underlying them are rearranged during synapse elimination. Soc Neurosci Abstr 15:165.

Balice-Gordon RJ, Lichtman JW (1990a) Loss of synaptic sites during competitive synapse elimination is both rapid and saltatory. Soc Neurosci Abstr 16:456.

Balice-Gordon RJ, Lichtman JW (1990b) In vivo visualization of the growth of pre- and postsynaptic elements of mouse neuromuscular junctions. J Neurosci 10:894-908.

Balice-Gordon RJ, Lichtman JW (1991) The plasticity and stability of neuromuscular synapses in living mice. In: Restorative neurology, Vol 5, Plasticity of motoneuronal connections, peripheral and central (Wernig A, ed), pp 71-84. Berlin: Springer.

Balice-Gordon RJ, Breedlove SM, Bernstein S, Lichtman JW (1990) Neuromuscular junctions shrink and expand as muscle fiber size is manipulated: in vivo observations in the androgen-sensitive bulbocavernosus muscle of mice. J Neurosci 10:2660-2671.

Betz WJ, Bewick GS (1992) Optical analysis of synaptic vesicle recycling at the frog ncuromuscular junction. Science 255:200-203.

Brown AP, van Mier P, Lichtman JW (1992) Synaptic plasticity: photobleaching recovery in living animals demonstrates neurotransmitter receptor movement in the postsynaptic membrane. Am Assoc Neurol Surgeons Abstr 60:323-324.

Brown MC, Jansen J, Van Essen D (1976) Polyneuronal innervation of skeletal muscle in new-born rats and its elimination during maturation. J Physiol (Lond) 261:387-422.

Chen L, Folsom DB, Ko C-P (1991) The remodeling of synaptic extracellular matrix and its dynamic relationship with nerve terminals at living frog neuromuscular junctions. J Neurosci 11:2920-2930.

Chua CK, Balice-Gordon RJ, Lichtman JW (1990) Differential labeling of terminal arbors of multiple axons innervating the same target cell with Dil and a new lipophilic tracer, 4-Di-16-ASP. Soc Neurosci Abstr 16:1004.

Grinvald A (1985) Real-time optical mapping of neuronal activity: from single growth cones to the intact mammalian brain. Annu Rev Neurosci 8:263-306.

Grinvald A, Lieke E, Frostig RD, Gilbert CD, Wiesel TN (1986) Functional architecture of cortex revealed by optical imaging of intrinsic signals. Nature 324:361-364.

Herrera AA, Banner LR, Nagaya N (1990) Repeated in vivo observation of frog neuromuscular junctions; remodelling involves concurrent growth and retraction. J Neurocytol 19:85-99.

Hill RR, Robbins NJ, Fang Z-P (1991) Plasticity of pre- and postsynaptic elements of neuromuscular junctions repeatedly observed in living adult mice. J Neurocytol 20:165-182.

Hubel DH, Wiesel TN (1963) Receptive fields of cells in striate cortex of very young, visually incxpcrienced kittens. J Ncurophysiol 28 : 1041-1059.

Hubel DH, Wiesel TN (1970) The period of susceptibility to the physiological effects of unilateral eye closure in kittens. J Physiol (Lond) 206:419-436.

Jansen JKS, Fladby T (1990) The perinatal reorganization of the innervation of skeletal muscle in mammals. Prog Neurobiol 34:39-90.

Jordan CL, Letinsky MS, Arnold AP (1988) Synapse elimination occurs late in the hormone-sensitive levator and muscle of the rat. $\mathrm{J}$ Neurobiol 19:335-356.

LeVay S, Wiesel TN, Hubel DH (1980) The development of ocular dominance columns in normal and visually deprived monkeys. $\mathrm{J}$ Comp Neurol 19:1-51.

Lichtman JW (1977) The reorganization of synaptic connections in the rat submandibular ganglion during postnatal development. $J$ Physiol (Lond) 273:155-177.

Lichtman JW, Magrassi L, Purves D (1987) Visualization of motor nerve terminals over time in living mice. J Neurosci 7:1215-1222.

Lichtman JW, Sunderland WJ, Wilkinson RS (1989) High resolution imaging of synaptic structure with a simple confocal microscope. New Biol 1:75-82.

London JA, Cohen LB, Wu J-Y (1989) Optical recordings of the cortical response to whisker stimulation before and after the addition of an epileptogenic agent. J Neurosci 9:2182-2190.

Magrassi L, Purves D, Lichtman JW (1987) Fluorescent probes that stain living nerve terminals. J Neurosci 7:1207-1214.

Nyström B (1968) Postnatal development of motor nerve terminals in 'slow-red' and 'fast-white' cat muscles. Acta Neurol Scand 44:363383.

Oda K (1986) Motor innervation and acetylcholine receptor distribution of human extraocular muscle fibres. J Neurol Sci 74:125-133.

Purves D, Lichtman JW (1980) Elimination of synapses in the developing nervous system. Science 210:153-157.

Ravdin P, Axelrod D (1977) Fluorescent tetramethyl rhodamine derivatives of $\alpha$-bungarotoxin: preparation, separation, and characterization. Anal Biochem 80:585-592.

Redfern PA (1970) Neuromuscular transmission in new-born rats. J Physiol (Lond) 209:701-709.

Rich MM, Lichtman JW (1989a) In vivo visualization of pre- and postsynaptic changes during synapse elimination in reinnervated mouse muscle. J Neurosci 9:1781-1805.

Rich MM, Lichtman JW (1989b) Motor nerve terminal loss from degenerating muscle fibers. Neuron 3:677-688.

Rosenthal JL, Taraskevitch PS (1977) Reduction of multiaxonal in- 
nervation at the ncuromuscular junction of the rat during development. J Physiol (Lond) 270:299-310.

Slater CR (1982a) Postnatal maturation of nerve-muscle junctions in hindlimb muscles of the mouse. Dev Biol 94:11-22.

Slater CR (1982b) Neural influence on the postnatal changes in acetylcholine receptor distribution at nerve-muscle junctions in the mouse. Dev Biol 94:23-30.

Steinbach JH (1981) Developmental changes in acetylcholine receptors at rat skeletal neuromuscular junctions. Dev Biol 84:267-276.
Stollberg J, Fraser SE (1990) Local accumulation of acetylcholine receptors is neither necessary nor sufficient to induce cluster formation. J Neurosci 10:247-255.

Wigston DJ (1989) Remodeling of neuromuscular junctions in adult mouse soleus. J Neurosci 9:639-647.

Wigston D (1990) Repeated in vivo visualization of neuromuscular junctions in adult mouse lateral gastrocnemius. J Neurosci 10:17531761 . 